FTPI-MINN-07/16

UMN-TH-2603/07

May 2/2007

\title{
$Q$ Torus in $\mathcal{N}=2 \mathrm{SQED}$
}

\author{
S. Bolognesi ${ }^{\diamond}$ and M. Shifman ${ }^{\star}$ \\ ${ }^{\diamond}$ The Niels Bohr Institute, Blegdamsvej 17, \\ DK-2100, Copenhagen Ø, Denmark, and \\ University of Southern Denmark, DK-5230 Odense, Denmark \\ *William I. Fine Theoretical Physics Institute, \\ University of Minnesota, Minneapolis, MN 55455
}

\begin{abstract}
We construct "Flying Saucer" solitons in supersymmetric $\mathcal{N}=2$ gauge theory which is known to support BPS domain walls with a $\mathrm{U}(1)$ gauge field localized on its worldvolume. We demonstrate that this model supports exotic particle-like solitons with the shape of a torus. $Q$ tori, and also similar solitons of higher genera, are obtained by folding the domain wall into an appropriate surface. Nontrivial cycles on the domain wall worldvolume (handles) are stabilized by crossed electric and magnetic fields inside the folded domain wall. Three distinct frameworks are used to prove the existence of these "Flying Saucer" solitons and study their properties: the worldvolume description (including the Dirac-Born-Infeld action), the bulk theory description in the sigma-model limit, and the bulk theory description in the thin-edge approximation. In the sigma-model framework the $Q$ torus is shown to be related to the Hopf Skyrmion studied previously.
\end{abstract}




\section{Contents}

1 Introduction 1

2 The bulk theory: a brief review of known facts 4

3 Fields Exited on the Wall 11

3.1 Constant magnetic flux inside the wall . . . . . . . . . . 11

3.2 Time dependence of $\alpha$ : what does it mean? . . . . . . . . . . . 14

$3.3 Q$ wall in the thin-edge approximation ......... 17

$3.4 Q$ wall in the sigma-model limit . . . . . . . . . 18

4 cylinder 20

$4.1 \quad Q$ cylinder from the bulk-theory standpoint . . . . . . . 22

4.2 Implementation of the $Q$ cylinder in the sigma-model limit . . 24

4.3 Comparing the bulk-theory and sigma model approximations . 26

4.4 Twisting the cylinder . . . . . . . . . . . . . . 28

5 Twisted and $Q$ charged torus 29

$5.1 Q$ torus in the full bulk theory . . . . . . . . . . . 31

$5.2 Q$ torus in the sigma-model limit . . . . . . . . . . . 35

5.3 Comment on the cycles' stability . . . . . . . . . . 36

5.4 Interactions between $Q$ tori . . . . . . . . . . . . . 37

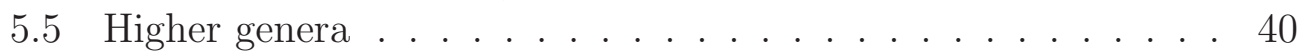

$5.6 Q$ charge radiation revisited ................ 42

6 The Dirac-Born-Infeld Action

6.1 Construction of the $Q$ torus from the DBI action . . . . . 4 45

7 Conclusions and open questions

References

\section{Introduction}

In this paper we show that the benchmark $\mathcal{N}=2$ gauge theory [1], which was known to support domain walls with a U(1) gauge field localized on the wall and the Abrikosov-Nielsen-Olesen flux tubes, also gives rise to exotic 
solitons whose topology is that of a torus (we also suggest the existence of arbitrary genus solitons). The stability is due to the existence of a number of conserved quantum numbers. One of them, $q$, is the $\mathrm{U}(1)$ charge on the surface; others $n, N, \ldots$ describe magnetic fluxes corresponding to various cycles. For instance, for a toroidal soliton, there are two cycles and, hence, two magnetic fluxes.

The general idea behind the solitons of the "Flying Saucer" type is as follows. We start from a "plane" domain wall (which is 1/2 BPS-saturated and topologically stable), and then fold it to make surfaces of arbitrary genera. These surfaces are closed and orientable. To stabilize all handles of the surfaces we let magnetic fluxes flow inside the wall. Each noncontractable cycle is characterized by its own flux. For instance, in the case of the $Q$ torus 1 , two magnetic fluxes must be nonvanishing. Magnetic fluxes by themselves are insufficient for complete stabilization. In addition to the magnetic fluxes the solitons under consideration must be $Q$ charged. The gauge $\mathrm{U}(1)$ symmetry is Higgsed in the bulk; therefore the electric field is screened in the bulk. However, a global U(1) survives providing for a possibility of $Q$ charging. Inside the wall we are in the Coulomb phase; therefore an electric field perpendicular to the wall surface can develop. It does develop if we endow the "Flying Saucer" soliton with the $Q$ charge. The combined action of the magnetic fluxes and the $Q$ charge leads to full stabilization.

We exploit three distinct (although not completely unrelated) frameworks to carry out our analysis: the worldvolume description (including that based on the Dirac-Born-Infeld action), the bulk theory description in the thinedge approximation and the bulk theory description in the sigma-model limit. All three considerations provide a proof of the existence of the $Q$ solitons and allow us to study some properties of the $Q$ torus - the $Q$ soliton on which we mostly focus in the present paper. In the sigma-model framework it becomes clear that the $Q$ torus is in fact related to the Hopf Skyrmion studied previously.

Our basic bulk theory is $\mathcal{N}=2$ supersymmetric $U(1)$ gauge theory with two "quark" hypermultiplets $Q^{A}, A=1,2$ and the Fayet-Iliopoulos term. Each hypermultiplet consists of two $\mathcal{N}=1$ chiral superfields, $Q^{A}$ and $\tilde{Q}_{A}$ $(A=1,2)$, with the electric charges $+1 / 2$ and $-1 / 2$, respectively. The quark

\footnotetext{
${ }^{1}$ We use a convention a la Coleman [15] and denote Q-solitons exitations that carry a charge of a conserved perturbative current.
} 
mass terms are introduced in the superpotential as follows

$$
\mathcal{W}_{m}=\sum_{A=1,2} m_{A} Q^{A} \tilde{Q}_{A}
$$

The existence of walls requires $m_{1} \neq m_{2}$. We will assume $m_{1,2}$ to be real and positive, and

$$
\Delta m \equiv\left|m_{1}-m_{2}\right| \ll m_{1,2} .
$$

Moreover, we will introduce the Fayet-Iliopoulos term. In Ref. [1] it was introduced through a superpotential; following [2] we will introduce it here through a $D$ term. These two methods reduce to each other identically $[3,4]$. An $\mathcal{N}=1$ chiral superfield $\mathcal{A}$ (with the lowest component $a$ ) - the $\mathcal{N}=2$ superpartner of the gauge field strength tensor $W$ - is also a part of our bulk theory.

Organization of the paper is as follows. In Sect. 2 we briefly review relevant aspects of the bulk theory. In Sect. 3 we consider the domain wall with fields ewxited on on its world volume. In 3.1 we explain how the magnetic flux can propagate inside the wall. (In the dual Polyakov language this is an electric field on the wall). In 3.2 we introduce a $Q$ charging procedure. We explain that the wall can be endowed with a conserved (global) U(1) charge which generates an electric field inside the wall perpendicular to the wall surface. (In the dual Polyakov language this is a magnetic field on the wall). Section 4 presents a $Q$ cylinder - a $Q$ wall folded in the form of a cylinder. Subsect. 4.1 treats the $Q$ cylinder from the bulk-theory standpoint, while in 4.2 the same object is analyzed in the sigma-model framework. These two complementary considerations are confronted in Subsect. 4.3. A new crucial element on the road to the $Q$ torus - twisting - is introduced in Subsect. 4.4. A concept design of the $Q$ torus is presented in Sect. 5. We discuss the $Q$ torus in the framework of the bulk theory in Subsect. 5.1. Then in 5.2 we continue this discussion in the sigma-model limit. Subsection 5.3 is a comment on stability of the $Q$ torus while Subsect. 5.4 addresses the issue of stability of the $Q$ solitons of higher genera. This discussion is continued in 5.5. Subsect. 5.6 is devoted to stability of the $Q$ torus with respect to leakage of the $Q$ charge in the bulk through radiation of elementary quanta. In Sect. 6 we use the Dirac-Born-Infeld action to further explore the $Q$ toric solitons in the framework of the worldvolume theory. Finally, Sect. 7 summarizes our conclusions and lists some open questions. 


\section{The bulk theory: a brief review of known facts}

The simplest model suitable for our construction is $\mathcal{N}=2$ SQED with two matter flavors. It was first used in the analysis of BPS domain walls supporting gauge fields on the wall surface in Ref. [1]. We refer the reader to [1] and the review paper [5] for a detailed description of the model. Here we will just outline basic features which will be necessary in what follows.

$\mathcal{N}=2$ supersymmetric $\mathrm{U}(1)$ gauge theory under consideration has two matter hypermultiplets $Q^{A}, A=1,2$. Each hypermultiplet consists of two $\mathcal{N}=1$ chiral superfields. We will introduce the following $\mathcal{N}=1$ chiral superfields:

$$
Q^{A} \text { and } \tilde{Q}_{A}
$$

$(A=1,2)$, with the electric charges $+1 / 2$ and $-1 / 2$, respectively. The quark mass terms are introduced via the superpotential,

$$
\mathcal{W}_{m}=\sum_{A=1,2} m_{A} Q^{A} \tilde{Q}_{A}
$$

The bosonic part of the action of this theory is

$$
\begin{aligned}
& S=\int d^{4} x\left\{-\frac{1}{4 g^{2}} F_{\mu \nu}^{2}+\frac{1}{g^{2}}\left|\partial_{\mu} a\right|^{2}+\bar{\nabla}_{\mu} \bar{q}_{A} \nabla_{\mu} q^{A}+\bar{\nabla}_{\mu} \tilde{q}_{A} \nabla_{\mu} \overline{\tilde{q}}^{A}\right. \\
& \left.-\left[\frac{g^{2}}{8}\left(\left|q^{A}\right|^{2}-\left|\tilde{q}_{A}\right|^{2}-\xi\right)^{2}+\frac{g^{2}}{2}\left|\tilde{q}_{A} q^{A}\right|^{2}+\frac{1}{2}\left(\left|q^{A}\right|^{2}+\left|\tilde{q}^{A}\right|^{2}\right)\left|a+\sqrt{2} m_{A}\right|^{2}\right]\right\},
\end{aligned}
$$

where

$$
\nabla_{\mu}=\partial_{\mu}-\frac{i}{2} A_{\mu}, \quad \bar{\nabla}_{\mu}=\partial_{\mu}+\frac{i}{2} A_{\mu}
$$

and $q, \tilde{q}$ are the scalar fields from $Q, \tilde{Q}$. Moreover, $\xi$ is the coefficient in front of the Fayet-Iliopoulos (FI) $D$ term, and $g$ is the $\mathrm{U}(1)$ gauge coupling. This is the simplest case which admits domain wall interpolating between two quark vacua.

There are two vacua in this theory: in the first vacuum

$$
a=-\sqrt{2} m_{1}, \quad q^{1}=\sqrt{\xi}, \quad q^{2}=0, \quad \tilde{q}=0,
$$


and in the second one

$$
a=-\sqrt{2} m_{2}, \quad q^{1}=0, \quad q^{2}=\sqrt{\xi}, \quad \tilde{q}=0 .
$$

The vacuum expectation value (VEV) of the field $\tilde{q}$ vanishes in both vacua, so we can disregard $\tilde{q}$ at the classical level.

Let us discuss the mass spectrum of light fields in both quark vacua. Consider for definiteness the first vacuum, Eq. (6). The spectrum can be obtained by diagonalizing the quadratic form in the above Lagrangian [1]. The result is as follows: one real component of the field $q^{1}$ is eaten up by the Higgs mechanism to become the third components of the massive photon. Three components of the massive photon, one remaining component of $q^{1}$, and four real components of the fields $\tilde{q}_{1}$ and $a$ form one long $\mathcal{N}=2$ multiplet ( 8 boson states +8 fermion states), with mass 2

$$
m_{\gamma}^{2}=\frac{1}{2} g^{2} \xi .
$$

The second flavor $q^{2}, \tilde{q}_{2}$ (which does not condense in this vacuum) forms one short $\mathcal{N}=2$ multiplet ( 4 boson states +4 fermion states), with mass $\Delta m$ which is heavier than the mass of the vector supermultiplet. In the second vacuum the mass spectrum is similar - the roles of the first and the second flavors are interchanged. The BPS domain wall which is a starting point of our construction interpolates between these two vacua. The wall solution is discussed in detail in [1]. We reiterate here qualitative features, see Fig. 1.

The mass parameters $m_{1}, m_{2}$ are assumed to be real. One can consider various limits for the ratio $|\Delta m| / \sqrt{\xi}$. First, we will assume

$$
|\Delta m| \gg g \sqrt{\xi} .
$$

In this approximation the domain wall has a well-defined three-layer structure: two edges whose thickness $\delta$ is small, $\delta \sim m_{\gamma}^{-1}$, and a wide middle domain whose thickness $d$ is large, $d \sim\left(\Delta m / m_{\gamma}\right) m_{\gamma}^{-1}$, see below. The thickness $d$, as well as the wall tension can be obtained as follows. First of all, one can neglect the edges $E_{1,2}$. This is a valid approximation, to be referred to as the thin-edge approximation. There are two contributions to the wall energy from the middle domain $M$ : the kinetic energy of the $a$ field $|\Delta a|^{2} /\left(g^{2} d\right)$

\footnotetext{
${ }^{2}$ The constraint (9) is equivalent to $\Delta m \gg m_{\gamma}$.
} 
and the loss of energy in the Coulomb vacuum $q=\tilde{q}=0$ in the middle of the wall, $g^{2} \xi^{2} d / 8$. We must minimize the expression for the wall tension

$$
T_{\mathrm{w}}=\frac{2(\Delta m)^{2}}{g^{2} d}+\frac{g^{2} \xi^{2} d}{8}
$$

with respect to $d$, which yields

$$
d=\frac{4 \Delta m}{g^{2} \xi}=\frac{2 \Delta m}{m_{\gamma}^{2}},
$$

and

$$
T_{\mathrm{w}}=\xi \Delta m .
$$

Although at first sight Eq. (12) might seem approximate, in fact it is exact [1].

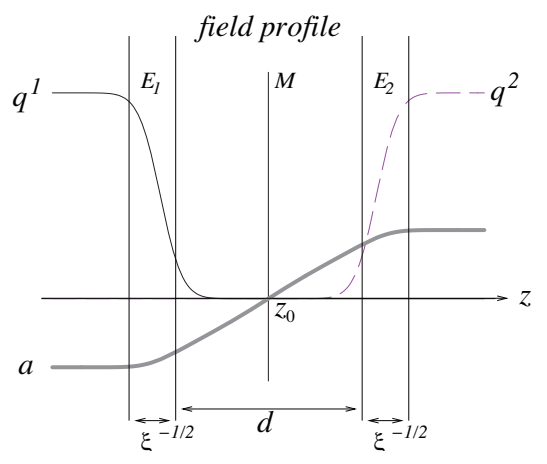

Figure 1: Internal structure of the domain wall: two edges (domains $E_{1,2}$ ) of the width $\sim(g \sqrt{\xi})^{-1}$ are separated by a broad middle band (domain $M$ ) of the width $d$, see Eq. (111).

A crucial feature of the domain wall solution [1] is the occurrence of a modulus - a collective coordinate characterizing the wall - of an angular type. The origin of this collective coordinate $\alpha$ can be explained as follows. The bulk theory at hand has in fact two $U(1)$ symmetries, corresponding to independent rotations of the first and second flavors (see Table 1). One of them is gauged, another global. The global symmetry is spontaneously broken on the given wall solution. That's why a massless phase field $\alpha$, a relative phase between two flavors in the two respective vacua, is trapped on the wall. The $\alpha$ modulus supplements an obvious collective coordinate, the wall center $z_{0}$. 
In what follows we will also consider the limit opposite to that in Eq. (9), the so-called sigma-model limit [6-8],

$$
\Delta m \ll g \sqrt{\xi},
$$

In this limit the "photonic" supermultiplet becomes heavier than that of the second flavor field. Therefore, it can be integrated out. Then we are left with the theory of (nearly) massless moduli $q^{2}, \tilde{q}_{2}$, which interact through a nonlinear sigma model with the Kähler term corresponding to the EguchiHanson metric. The manifold parametrized by these (nearly) massless fields is obviously four-dimensional. The vacua discussed above lie at the base of this manifold. Therefore, in considering the domain wall solutions in the sigma-model limit $\Delta m \rightarrow 0$ [6-8] one can limit oneself to the base manifold, which is, in fact, a two-dimensional sphere. Classically, it is sufficient to consider the domain wall in the $\mathrm{CP}(1)$ model deformed by a twisted mass term (related to a nonvanishing $\Delta m$ ). This was first done in [7]. A more general analysis of the domain walls on the Eguchi-Hanson manifold can be found in [9]. For our purposes it will be sufficient to limit ourselves to the twisted-mass deformed CP(1) model.

The bosonic part of the Lagrangian of the sigma model is

$$
\mathcal{L}=\frac{1}{2} \partial_{\mu} \vec{\varphi} \cdot \partial^{\mu} \vec{\varphi}-\frac{1}{2}(\Delta m)^{2}\left(\xi-\varphi_{3}^{2}\right), \quad \vec{\varphi} \cdot \vec{\varphi}=\xi .
$$

Using the stereographic projection (see e.g. the review paper [10]) we can make the following change of coordinates:

$$
u=\frac{\varphi_{1}+i \varphi_{2}}{\sqrt{\xi}-\varphi_{3}} .
$$

As a result, our sigma model takes the form

$$
\mathcal{L}=\xi\left\{\frac{\partial_{\mu} \bar{u} \partial_{\mu} u}{(1+\bar{u} u)^{2}}-(\Delta m)^{2} \frac{\bar{u} u}{(1+\bar{u} u)^{2}}\right\} .
$$

Note that in the sigma-model limit only two fundamental parameters are left: $\Delta m$ and $\xi$. Information about the coupling constant $g$ is lost. In fact, this limit is sometimes called the "strong coupling limit" since the condition (13) can be reached by just sending the coupling constant $g$ to infinity.

We can readily obtain the BPS domain wall in the sigma-model approximation too. In Fig. 2 we display the sigma models target space, a sphere $\mathbf{S}^{2}$. 
The two vacua - the minima of the potential term - are the north and the south poles, corresponding to $u=\infty$ and $u=0$, respectively. The domain wall "trajectory" is an arc interpolating between the two poles. From Fig. 2 the origin of the $\mathrm{U}(1)$ modulus in this approximation is quite clear: The wall "trajectory" can run along any meridian. For a pedagogical review see [11].

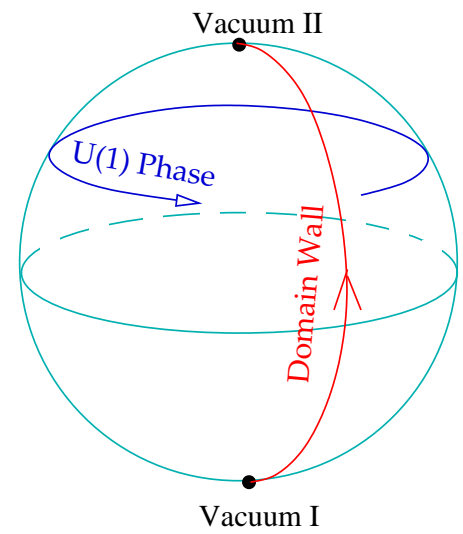

Figure 2: The sigma model target space, the domain wall and its $U(1)$ modulus. The vacua $I$ and $I I$ correspond to $u=0$ and $u=\infty$, respectively.

To get a quantitative description of the domain wall in the sigma-model approximation we perform the Bogomol'nyi completion of the sigma-model Lagrangian,

$$
T=\int d z \xi\left\{\left|\frac{\partial_{z} u \pm \Delta m u}{1+\bar{u} u}\right|^{2} \mp \Delta m \frac{\partial}{\partial z}\left(\frac{1}{1+\bar{u} u}\right)\right\} .
$$

We observe that the BPS equation

$$
\partial_{z} u=\mp u
$$

is holomorphic; therefore, the wall center modulus $z_{0}$ is complexified,

$$
u=e^{\mp \Delta m\left(z-z_{0}\right)+i \alpha},
$$

Any constant phase $\alpha$ is allowed. The full derivative term in Eq. (17) implies that the wall tension

$$
T_{\mathrm{w}}=\xi|\Delta m|,
$$




\begin{tabular}{|c|c|c|c|c|}
\hline Gauged U(1) & $q^{1} \rightarrow e^{i \alpha / 2} q^{1}$ & $q^{2} \rightarrow e^{i \alpha / 2} q^{2}$ & $\tilde{q}_{1} \rightarrow e^{-i \alpha / 2} \tilde{q}_{1}$ & $\tilde{q}_{2} \rightarrow e^{-i \alpha / 2} \tilde{q}_{2}$ \\
\hline Global U(1) & $q^{1} \rightarrow e^{i \alpha / 2} q^{1}$ & $q^{2} \rightarrow e^{-i \alpha / 2} q^{2}$ & $\tilde{q}_{1} \rightarrow e^{-i \alpha / 2} \tilde{q}_{1}$ & $\tilde{q}_{2} \rightarrow e^{i \alpha / 2} \tilde{q}_{2}$ \\
\hline
\end{tabular}

Table 1: Two U(1) symmetries of the bulk theory.

in full accord with Eq. (12).

The fact that each domain wall has two bosonic collective coordinates its center and the phase - in the sigma-model limit was noted in [7].

Now let us describe the domain wall dynamics from another point of view: that of the $2+1$ effective theory on the wall worldvolume. The wall solution is characterized by two collective coordinates, the position of the wall center $z_{0}$ and th phase $\alpha$. In the effective low-energy theory on the wall they become scalar fields of the worldvolume $(2+1)$-dimensional theory, $z_{0}(t, x, y)$ and $\alpha(t, x, y)$, respectively. The target space of the field $\alpha$ is $S_{1}$.

Let us make the wall collective coordinates $z_{0}$ and $\alpha$ (together with their fermionic superpartners) slowly varying fields depending on $x_{n}(n=0,1,2)$. Since $z_{0}\left(x_{n}\right)$ and $\alpha\left(x_{n}\right)$ correspond to zero modes of the wall, they have no potential terms in the worldsheet theory. It is easy to derive the $z_{0}$ kinetic term,

$$
S_{2+1}^{z_{0}}=\frac{T_{\mathrm{w}}}{2} \int d^{3} x\left(\partial_{n} z_{0}\right)^{2},
$$

while the derivation of the normalization factor for the kinetic term for $\alpha$ is rather tedious, albeit straightforward [1],

$$
S_{2+1}^{\alpha}=\frac{\xi}{\Delta m} \int d^{3} x \frac{1}{2}\left(\partial_{n} \alpha\right)^{2} .
$$

As is well known from Polyakov's work [12], the compact scalar field $\alpha(t, x, y)$ can be dualized into a $(2+1)$-dimensional Abelian gauge field. Namely,

$$
F_{n m}^{(2+1)}=\frac{e^{2}}{2 \pi} \varepsilon_{n m k} \partial^{k} \alpha,
$$

where the $(1+2)$-dimensional coupling $e^{2}$ is

$$
e^{2}=4 \pi^{2} \frac{\xi}{\Delta m}
$$


Finally one gets the following effective low-energy theory of the moduli fields on the wall:

$$
S_{2+1}=\int d^{3} x\left\{\frac{T_{\mathrm{w}}}{2}\left(\partial_{n} z_{0}\right)^{2}+\frac{1}{4 e^{2}}\left(F_{n m}^{(2+1)}\right)^{2}+\text { fermion terms }\right\} .
$$

The emergence of the gauge field on the wall is easy to understand. Let us start from a magnetic monopole in the bulk. Since in the bulk the theory is fully Higgsed, the magnetic flux must be squeezed inside an ANO flux tube [13] which starts on the monopole and ends on the wall (it is oriented perpendicular to the wall). Inside the wall the $q$ condensates vanish, the $\mathrm{U}(1)$ gauge group is restored, and the magnetic flux spreads freely. In the Polyakov dual language the magnetic flux lines spread inside the wall are the electric flux lines on the wall. A vortex in $\alpha$ corresponds to a charge source for the electric field on the wall.

The fermion content of the worldvolume theory is given by two threedimensional Majorana spinors, as is required by $\mathcal{N}=2$ in three dimensions (four supercharges). The full worldvolume theory is a $U(1)$ gauge theory in $(2+1)$ dimensions, with four supercharges. The Lagrangian (25) and the corresponding superalgebra can be obtained by reducing four-dimensional $\mathcal{N}=1$ SQED (with no matter) to three dimensions. The field $z_{0}$ in (25) is the $\mathcal{N}=2$ superpartner of the gauge field $A_{n}$. To make it more transparent we make a rescaling, introducing a new field

$$
a_{2+1}=2 \pi \xi z_{0} .
$$

In terms of $a_{2+1}$ the action (25) takes the form

$$
S_{2+1}=\int d^{3} x\left\{\frac{1}{2 e^{2}}\left(\partial_{n} a_{2+1}\right)^{2}+\frac{1}{4 e^{2}}\left(F_{m n}^{(2+1)}\right)^{2}+\text { fermions }\right\} .
$$

The gauge coupling constant $e^{2}$ has dimension of mass in three dimensions.

The lightest massive excitations of the wall have mass of order of the inverse thickness of the wall $1 / d$, see (11). Thus the dimensionless parameter that characterizes the coupling strength in the worldvolume theory is $e^{2} d$,

$$
e^{2} d=\frac{16 \pi^{2}}{g^{2}}
$$

This can be interpreted as a feature of the bulk-wall duality: the weak coupling regime in the bulk theory corresponds to strong coupling on the wall and vice versa [1]. 
To conclude this section let us briefly summarize various limits considered previously in this problem, and their range of validity.

\section{Thin-edge approximation :}

In this approximation the mass of the scalar fields is much larger than the photon mass, $|\Delta m| \gg g \sqrt{\xi}$. The domain wall has a three-layer structure and its properties can be analyzed by virtue of very simple equations.

\section{Sigma-model limit :}

In this approximation the mass of the scalar field is much smaller than the photon mass, $|\Delta m| \ll g \sqrt{\xi}$. The heavy fields can be integrated out, and what is left is a non-linear sigma model with the target space $\mathbf{S}^{2}$ and a potential which leaves the north and the south poles as the only vacua of the theory.

\section{Worldvolume effective action :}

Excitations of the wall moduli can be studied, in the low-energy limit, by virtue of an effective Lagrangian on the domain wall worldvolume.

Here by low energies we mean energies much lower than $\Delta m$ and $g \sqrt{\xi}$.

We will deal with these limits wherever appropriate in the subsequent parts of the paper.

\section{$3 \quad$ Fields Exited on the Wall}

We now consider exited states of the domain wall. First switching on a magnetic field and then an electric field.

\subsection{Constant magnetic flux inside the wall}

In Ref. [14] it was noted that moduli fields in supersymmetric theories have a new type of stable "vacuum" solutions which preserve a part of supersymmetry. In the case of the phase modulus this solution can be presented as

$$
\alpha=a x
$$


where $a$ is a constant of dimension of mass and $x$ is one of two coordinates on the domain wall. It is clear that the solution (29) satisfies the equation

$$
\left(\frac{\partial^{2}}{\partial x^{2}}+\frac{\partial^{2}}{\partial y^{2}}\right) \alpha=0 \text {. }
$$

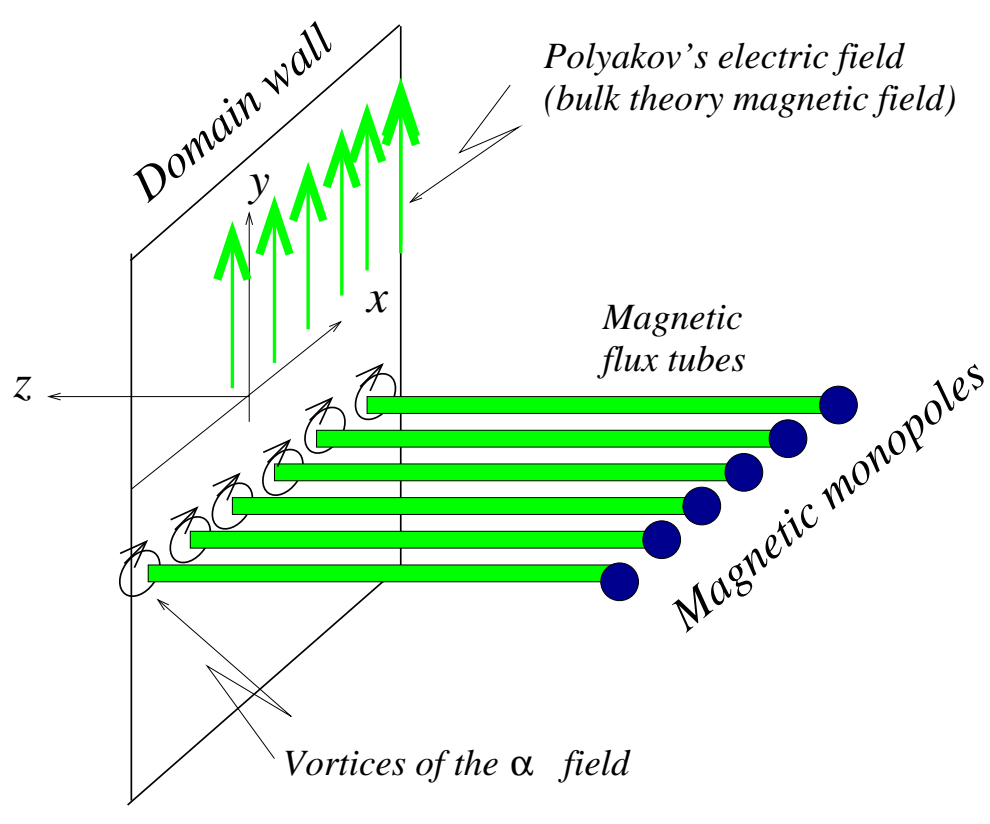

Figure 3: In the limit of vanishing distance between the magnetic flux tubes (perpendicular to the wall) the field $\alpha$ on the wall becomes linear in $x$. This is equivalent to a constant Polyakov electric field on the wall surface aligned in the $y$ direction. From the bulk point of view we have a constant magnetic field aligned in the $y$ direction propagating inside the wall parallel to its surface.

In the Polyakov language this solution corresponds to a $F_{0 y}^{(2+1)}$ component of the dual field strength tensor (23). In the bulk-theory language we describe in this way a constant magnetic field inside the wall directed along the $y$ axis. Figure 3 illustrates the validity of this interpretation. The bulk part is depicted only with the purpose of making clear the physical basis of the construction. After this is done, one can eliminate it and consider an infinite wall stretched in the $x y$ plane with a constant magnetic flux propagating inside the wall (Fig 4). The value of the flux per length $\ell$ in the $x$ direction is $2 a \ell$. The factor 2 here is in one-to-one correspondence with way we define 
the electric charge in Eq. (5) , i.e. the coefficient $\frac{1}{2}$ in front of $A_{\mu}$. In this definition the magnetic flux of the magnetic monopole is $4 \pi$.

The absolute value of the magnetic field inside the wall is

$$
|\vec{B}|=2 d^{-1} a,
$$

where for definiteness $a$ is assumed to be positive.

The topological defect depicted in Fig. 4 is stable - the vacua on the right- and left-hand sides of the wall are distinct. The tension of this defect is

$$
T_{\mathrm{w} \mathrm{B}}=\xi \Delta m\left[1+\frac{a^{2}}{2(\Delta m)^{2}}\right],
$$

where the subscript $\mathrm{B}$ corresponds to the presence of the magnetic field $B$ inside the wall. We have to assume that $a / \Delta m \ll 1$. We will discuss later how this limitation can be lifted.

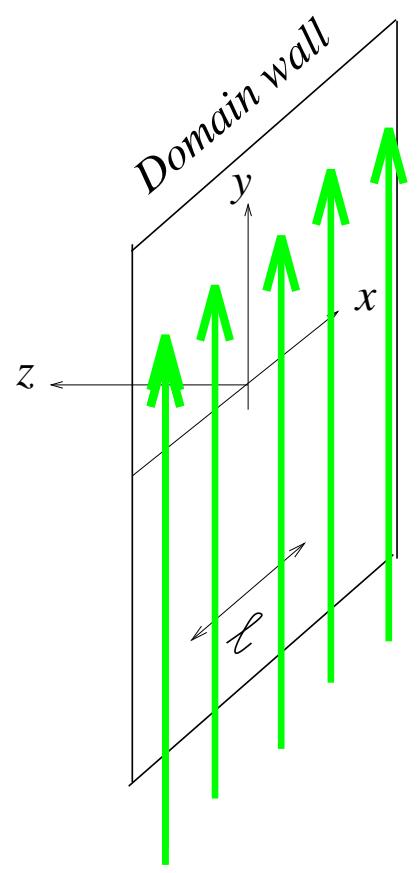

Figure 4: A domain wall with a constant magnetic flux propagating inside the wall. The value of the magnetic flux per length $\ell$ in the $x$ direction is $2 a \ell$.

In the case of the infinite wall, the magnetic flux is not quantized. However, the flux quantization does take place if we compactify the horizontal 
direction, making a cylinder from the wall at hand. That's what we will do below.

\subsection{Time dependence of $\alpha$ : what does it mean?}

From the point of view of the classical equation of motion and stability a linear function in $t$ is as good a solution as (29). Assume that

$$
\alpha=\omega t
$$

where $\omega$ is a constant of dimension of mass. In the Polyakov language this means that an $x y$ component of the dual $(2+1)$-dimensional field is generated,

$$
F_{12}^{(2+1)}=2 \pi \frac{\xi \omega}{\Delta m}
$$

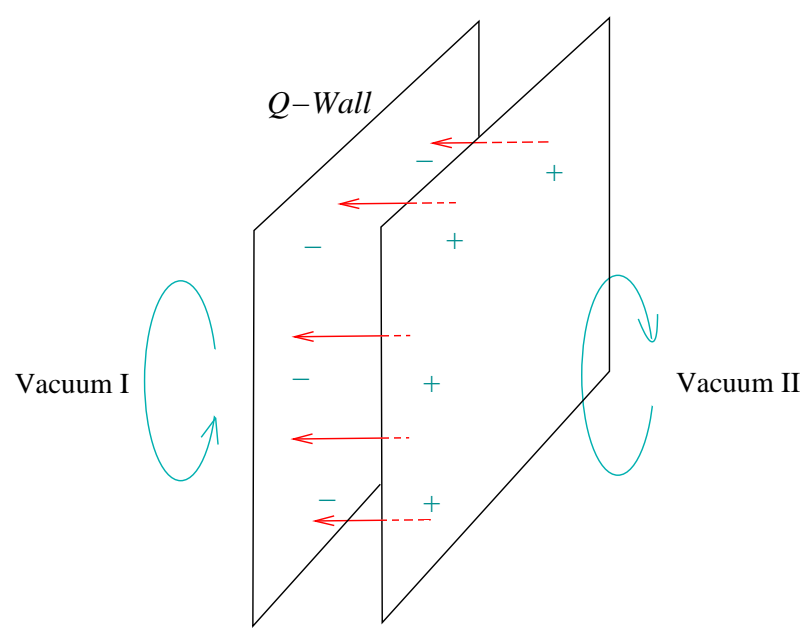

Figure 5: Q charged domain wall. The electric field inside the wall (it is perpendicular to the wall surface) is denoted by arrows.

The $x y$ component is the Polyakov dual magnetic field (which is a scalar with respect to rotations in the $x y$ plane). In the bulk theory this corresponds to an electric field perpendicular to the wall surface, see Fig. 5. If $\omega \neq 0$, then the tension of the wall with the electric and magnetic fields inside is

$$
T_{\mathrm{w} \mathrm{BE}}=\xi \Delta m\left[1+\frac{a^{2}}{2(\Delta m)^{2}}+\frac{\omega^{2}}{2(\Delta m)^{2}}\right] \text {, }
$$


where the subscript E reminds us of the electric field $E$ inside the wall. Equation (34) implies that the absolute value of the electric field inside the wall is

$$
|\vec{E}|=2 d^{-1} \omega \text {. }
$$

So far we focused on the worldvolume theory on the wall and interpreted the "vacua" with $\alpha$ linearly dependent on $t, x$ in terms of $\vec{B}$ and $\vec{E}$ inside the wall. Let us ask ourselves about the picture of this phenomenon viewed from outside, from the bulk.

The bulk theory (4) has two U(1) symmetries: one global, one local. The local $\mathrm{U}(1)$ is realized in the Higgs mode, i.e. there is a charge condensate in the bulk and no massless gauge bosons. This is the reason why the electric field inside the wall must be perpendicular to its surface. The charge $Q$ corresponding to the conserved global U(1) which emerges after the theory is Higgsed in the bulk can be chosen on the wall surface at will as long as it does not change with time. If $\omega \neq 0$ the domain wall carries a nonvanishing density of $Q$,

$$
Q / \text { Area }=\frac{\xi \omega}{2 \Delta m}
$$

where $\omega$ is assumed to be positive. $Q$-charged solitons were invented by Coleman, who considered a particular type [15] currently known as $Q$ balls. Generalizations were discussed in $[16,17]$.

Elementary excitations of the vacuum carry a nonvanishing $Q$ charge too. For instance, to the right of the wall, far away from it (Fig. 1) one can always require $q^{2}$ to be real. The quantum of $q^{1}$ carries a phase, however, and, as a result, will have $Q= \pm 1$. The question is what prevents $Q$ from leaking from the wall into the bulk through particle emission.

The answer to this question seems to be rather straightforward. A unit charge inside the wall edge is lighter than a particle with the same charge propagating in the vacuum. In other words, the work function is high. Indeed, the area of the segment of the wall with the unit $\mathrm{U}(1)$ charge is

$$
A \sim \frac{\Delta m}{\xi \omega} .
$$

The excess of energy corresponding to this segment is

$$
\Delta E=\Delta T A \sim \omega
$$


where we used Eqs. (34) and (37). This is to be compared with the mass of the $Q=1$ particle, $\Delta m$ (Sect. 2). Stability is guaranteed provided

$$
\omega \ll \Delta m .
$$

Stability of solitons of this type in theories with a conserved U(1) charge is well studied in supersymmetric models. For a recent analysis of twodimensional $\mathcal{N}=2$ sigma model with twisted mass see [18]. In this model there is a domain in the parameter space in which $Q$ kinks cannot emit elementary $Q$ charged excitations because such emission is energetically forbidden. A curve of marginal stability separates this domain from that where the tower of $Q$ charged kinks decays.

A remark is in order here regarding " $Q$ charging" from the standpoint of the bulk theory. Examining the Lagrangian (4) we see that the $Q$ kink is a soliton with four excited fields: three scalar fields $q_{1}, q_{2}$, a, and the time component of the gauge potential $A_{t}$. Table 2 gives a summary of the corresponding profile functions, with the appropriate boundary conditions.

\begin{tabular}{|c|c|c|}
\hline & $x_{3} \rightarrow-\infty$ & $x_{3} \rightarrow+\infty$ \\
\hline$q_{1}\left(x_{3} ; t\right)$ & $\sqrt{\xi} e^{i \frac{\omega}{2} t}$ & 0 \\
\hline$q_{2}\left(x_{3} ; t\right)$ & 0 & $\sqrt{\xi} e^{-i \frac{\omega}{2} t}$ \\
\hline$a\left(x_{3}\right)$ & $-\sqrt{2} m_{1}$ & $-\sqrt{2} m_{2}$ \\
\hline$A_{t}\left(x_{3}\right)$ & $\omega$ & $-\omega$ \\
\hline
\end{tabular}

Table 2: Profile functions relevant to the $Q$-kink soliton in the bulk theory. Note that $x_{3} \equiv z$.

$Q$ charging is attained in two steps. First we take the domain wall and give it a global $\mathrm{U}(1)$ phase rotation $e^{i \omega t}$. However, this step alone would result in a divergence in the wall tension due to the time derivatives of the type $\partial_{t} q$. The second step is to compensate the time dependence of $q$ 's far away from the wall by introducing a gauge potential $A_{t}$.

A subtle point here is that the $\mathrm{U}(1)$ gauge transformation is different from the global U(1). They can be identified, however (in two distinct ways depending on whether we are to the left or to the right from the wall). 
Namely, at $x_{3} \rightarrow-\infty$ we must have $A_{t} \rightarrow \omega$ while at $x_{3} \rightarrow+\infty$ we must have $A_{t} \rightarrow-\omega$. The sign of the gauge potential is different at two extremities. Step two thus cures the divergence of the wall tension but it leaves a trace. $A_{t}$ is in fact pure gauge only far away from the wall. In wall's interior an electric field is generated.

The $Q$ wall in the full bulk theory can be analyzed with more precision. We refer the reader e.g. to Ref. [19] for the Bogomol'nyi completion and derivation of the first-order differential equations.

Below we use the bulk theory picture in order to generalize Eq. (34) and other similar expressions to larger values of the $Q$ charge.

\section{3 $Q$ wall in the thin-edge approximation}

In the thin-edge approximation the $Q$ wall can be viewed as an electrostatic capacitor. Let us assume there is a positive charge density $+\sigma$ on one edge (say, on the left) and a negative charge density $-\sigma$ on the other edge. 3

Inside the capacitor, between the two edges, we have an electric field $E_{z}=\sigma$. The wall tension is now given by a sum of three terms,

$$
T_{Q-\text { wall }}(d)=\frac{2(\Delta m)^{2}}{g^{2} d}+\frac{g^{2} \xi^{2}}{8} d+\frac{\sigma^{2}}{2 g^{2}} d .
$$

Equation (40) replaces Eq. (10) where the electric field inside the wall is not included. Inclusion of the electric field is equivalent to the substitution

$$
\xi^{2} \rightarrow \xi^{2}+\frac{4 \sigma^{2}}{g^{4}}
$$

Minimizing with respect to $d$ in the same way as above we obtain

$$
d_{Q-\text { wall }}=\frac{4 \Delta m}{g^{2} \xi}\left(1+\frac{4 \sigma^{2}}{g^{4} \xi^{2}}\right)^{-1 / 2}
$$

and

$$
T_{Q-\text { wall }}=\Delta m \xi\left(1+\frac{4 \sigma^{2}}{g^{4} \xi^{2}}\right)^{1 / 2}
$$

\footnotetext{
${ }^{3}$ In our conventions the electromagnetic Hamiltonian $\mathcal{H}=\left(2 g^{2}\right)^{-1}\left(\vec{E}^{2}+\vec{B}^{2}\right)$. Then the Gauss' theorem implies that the charge $=g^{-1} \int_{\mathcal{S}_{R}} d^{2} S_{i} E_{i}$. The electric field $E$ in the capacitor is $|E|=\sigma$. The minimal charge of the quantum of the $q$ field is $1 / 2$, see Eq. (5). Thus, in our normalization the unit of the $\mathrm{U}(1)$ charge is in fact $g^{2} / 2$.
} 
One can see that Eq. (10) presents a small- $\sigma$ expansion of Eq. (43) provided one identifies

$$
\omega=2 \sigma \frac{\Delta m}{g^{2} \xi} .
$$

We already know that at small $\omega$ the $Q$ charged wall is stable with respect to "leakage" of the U(1) charge outside in the form of emission of quanta of the $q$ fields. Now we can check that this remains valid for large $Q$ charges as well. In fact, in the limit $\sigma \rightarrow \infty$ the $Q$ wall becomes marginally stable (Fig. 66).

Indeed, at large $\sigma$ the wall tension becomes linear in $\sigma$,

$$
T_{Q-\text { wall }}=\frac{2}{g^{2}} \Delta m \sigma
$$

Let us pick up an area $A$ such that a change $\delta \sigma$ on this area could produce a quantum of the unit charge. Given our normalization $A \delta \sigma=g^{2} / 2$. The corresponding variation of the energy of the wall is

$$
\delta E=A \delta T_{Q-\text { wall }}=A \frac{2}{g^{2}} \Delta m \delta \sigma=\Delta m .
$$

This is precisely the mass of a single $q$ quantum.

\section{4 $Q$ wall in the sigma-model limit}

Alternatively we can consider the $Q$ wall in the sigma model approximation. To obtain the wall solution in the sigma model we perform the following completion procedure:

$$
\begin{aligned}
T & =\int d z \xi\left\{\frac{\partial_{t} \bar{u} \partial_{t} u}{(1+\bar{u} u)^{2}}+\frac{\partial_{z} \bar{u} \partial_{z} u}{(1+\bar{u} u)^{2}}+(\Delta m)^{2} \frac{\bar{u} u}{(1+\bar{u} u)^{2}}\right\} \\
& =\int d z \xi\left\{\frac{\left[\partial_{t} u \pm i(\Delta m)(\sinh b) u\right]\left[\partial_{t} \bar{u} \pm i(\Delta m)(\sinh b) \bar{u}\right]}{(1+\bar{u} u)^{2}}\right. \\
& +\left|\frac{\partial_{z} u \pm(\Delta m)(\cosh b) u}{1+\bar{u} u}\right|^{2} \pm i(\Delta m)(\sinh b) \partial_{t}\left[\frac{1}{1+\bar{u} u}\right] \\
& \left. \pm(\Delta m)(\cosh b) \partial_{z}\left[\frac{1}{1+\bar{u} u}\right]\right\}
\end{aligned}
$$


where $b$ is a constant. The solution takes the form (for the lower choice of the sign)

$$
u=e^{i(\Delta m)(\sinh b) t} e^{(\Delta m)(\cosh b)\left(z-z_{0}\right)}
$$

while the tension

$$
T_{\sigma-\text { model }}=\xi(\Delta m)(\cosh b) .
$$

To compare with our previous results it is convenient to normalize $b$ as

$$
\omega=(\Delta m)(\sinh b)
$$

where the frequency $\omega$ was introduced earlier. Then

$$
T_{\sigma-\text { model }}=\xi \sqrt{(\Delta m)^{2}+\omega^{2}},
$$

which at small $\omega$ reduces to the $\omega$ dependent part of Eq. (34). Moreover, the wall solution takes the form

$$
u=e^{i \omega t} e^{\sqrt{(\Delta m)^{2}+\omega^{2}}\left(z-z_{0}\right)} .
$$

One can readily see that we get the same expression for the $Q$ wall tension as in the thin-edge approximation (cf. Eq. (44)). Therefore, the same stability argument applies. The marginal stability at large $Q$ is clearly seen from Fig. 6.

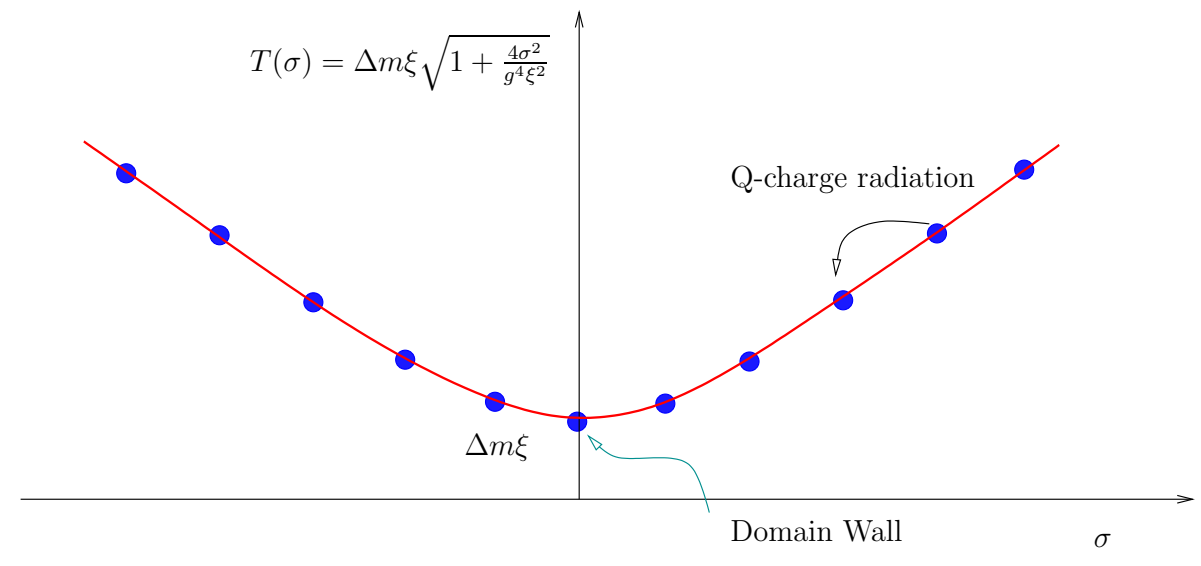

Figure 6: The $\mathrm{Q}$ wall tension vs. $\sigma$. In our normalization the elementary unit of the $\mathrm{U}(1)$ charge is $\frac{g^{2}}{2}$. The $\mathrm{Q}$ wall is stable with respect to radiation of $q$ quanta. It becomes asymptotically marginally stable at $\sigma \rightarrow \infty$. 


\section{$4 \quad Q$ cylinder}

As was mentioned, the basic wall depicted in Fig. 1 is absolutely stable as long as it is flat and its area is infinite. This stability does not require electric or magnetic fluxes which may or may not be introduced with no impact on stability.

Our strategy is to start from this flat wall, and bend/fold it in an appropriate way to get solitons with a more complicated geometry without loosing stability. A natural starting point is cylindrical geometry.

If we try to fold the "empty" wall (no fluxes) in the form of a cylinder (Fig. 7 a) it becomes unstable. Nothing prevents it from shrinking in the radial direction until the vacuum $I$ is completely "squeezed out" so that the wall-cylinder decays into a large number of elementary excitations in the vacuum $I I$. Of course, if the radius of the cylinder is much larger than the thickness of the wall — and we will assume this to be the case — the process of shrinkage will be slow until the field configuration at hand becomes a "thin cylinder," i.e. the one whose radius is only slightly larger than the wall thickness.

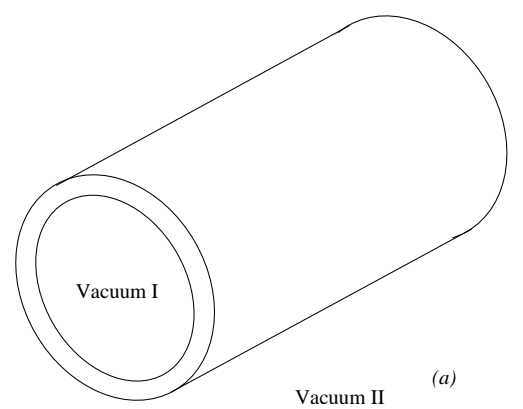

Figure 7: Folding the domain wall.

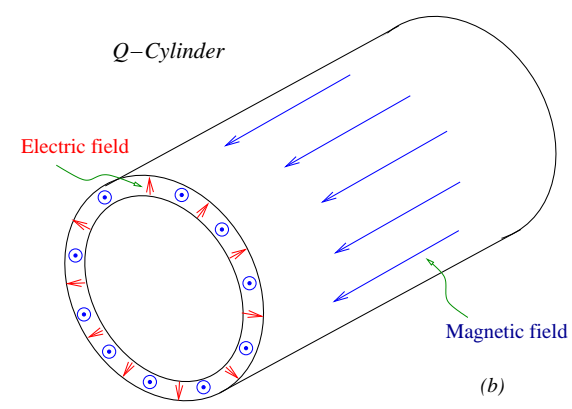

(a) Making a cylinder from the basic ("empty") wall; (b) Magnetic and electric fields inside the wall.

Can one stabilize the cylinder? The first thought that comes to one's mind is to let the magnetic flux propagate inside the cylinder in the direction of its axis. Then disintegration of the cylinder via decays into elementary excitations in the vacuum $I I$ is impossible since the magnetic flux is conserved. However, the magnetic flux conservation does not prevent the cylinder from passing into the ANO strings [13] with the same value of the total magnetic flux, plus elementary excitations. Whether or not the cylinder with the magnetic field trapped inside the wall is stable with respect to such decays is a 
dynamical question. Note that in the cylindrical geometry the magnetic flux gets quantized.

Assume that the radius of the cylinder is very large so that locally a flat wall approximation is applicable. Then the phase field $\alpha$ will continue to provide an adequate description of the worldsheet dynamics. However, globally the change of $\alpha$ in the compactified direction must be of the form $2 \pi n$ with an integer $n$. If the circumference of the cylinder is $L$, this implies

$$
\alpha=2 \pi n \frac{x}{L} .
$$

Correspondingly, the magnetic flux trapped inside the wall is $4 \pi n$, i.e. that of $n$ magnetic monopoles. At $n=1$ the flux is the same as the ANO string. The cylindrical wall will be stable against decays into the ANO string(s) plus elementary excitations provided the $n=1$ cylinder tension is less than that of the ANO string. Unfortunately, this is not the case. For instance, in the sigma-model approximation one can readily get that the ratio of the above tensions is $\sqrt{2}$. The cylindrical wall with the magnetic flux is heavier than the ANO strings with the same flux. Intuitively this seems natural since the internal structure of the cylindrical wall is more complicated than that of the ANO string.

Thus, the simplest attempt to stabilize the wall-cylinder fails. This is not the end of the story, however. In addition to the magnetic field inside the wall, one can $Q$-charge it, i.e. introduce an electric field in the radial direction, as in Fig. 7b. For sufficiently large radius of the cylinder we still can write

$$
\alpha=2 \pi n \frac{x}{L}+\omega t
$$

where $x$ is the coordinate perpendicular to the cylinder axis (see Fig. 8).

The non-vanishing value of the $Q$ charge does make the wall-cylinder stable since the ANO strings are $Q$ neutral, which precludes the decay of the $Q$ charged wall-cylinder into the ANO strings plus elementary excitations. Elementary excitations are $Q$ charged, generally speaking, but as was discussed in Sect. 3.2, the leakage of the wall $Q$ charge into elementary excitations is energetically forbidden, at least as long as the wall is nearly flat.

The shrinkage of the cylinder in the radial direction due to the wall tension is stabilized by the magnetic field inside the wall. Indeed, let $\rho$ denote the cylinder radius (Fig. 8) and $4 \pi n$ the magnetic flux. Then the magnetic field

$$
|\vec{B}| \sim \frac{n}{\rho d}
$$




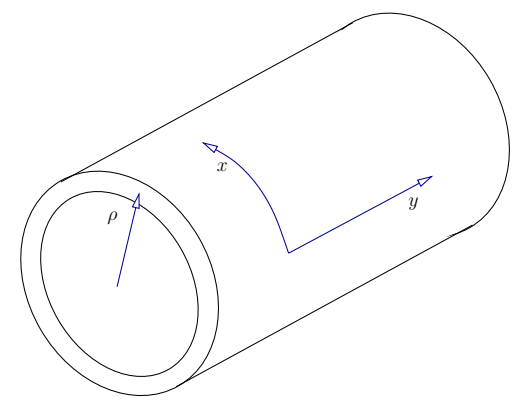

Figure 8: Geometry of the cylindrically folded wall.

where $d$ is given in Eq. (11). Thus, the energy stored in the magnetic field per unit length of the cylinder is

$$
\mathcal{E}_{B} \sim \frac{n^{2}}{g^{2} d \rho}
$$

A more detailed consideration of the $Q$ cylinder from the bulk-theory standpoint is given in Sect. 4.1 where we determine the critical values of $\rho$ and $d$ in terms of $Q$ and $n$ and formulate the condition of "thin cylinder walls" implying $\rho \gg d$.

\section{1 $Q$ cylinder from the bulk-theory standpoint}

Here we will parallel our discussion of the $Q$ wall in the thin-edge approximation carried out in Sect. 3.3 extending it to the $Q$ cylinder. The method we use is very similar to the one used in $[20,21]$ to study the large- $n$ limit of the Abrikosov-Nielsen-Olesen vortex. As in Sect. 4 we will assume the radius of the cylinder $\rho$ to much larger than the wall thickness $d$ (which, in turn, is much larger than the thickness of the edge layers, to be set to zero in our approximation). As in the $Q$ wall case, there are four distinct contributions to the $Q$ cylinder tension: (i) the loss of energy due to the Coulomb phase inside the cylinder wall; (ii) kinetic energy of the $a$ field, (iii) the energy of the magnetic field inside the cylinder wall; and (iv) the energy of the electric field. The corresponding expressions have a somewhat different form. Let us discuss them one by one. Our starting point will be Eq. (40).

(i) The loss of energy due to the Coulomb phase inside the cylinder wall per unit length of the cylinder can be obtained from the second term in 
Eq. (40),

$$
\frac{\pi}{4} g^{2} \xi \rho d .
$$

(ii) The kinetic energy of the $a$ field per unit length of the cylinder can be obtained from the first term in Eq. (40),

$$
\frac{4 \pi}{g^{2}}(\Delta m)^{2} \frac{\rho}{d} .
$$

(iii) The magnetic field energy per unit length is (see Eq. (561))

$$
\frac{4 \pi n^{2}}{g^{2}} \frac{1}{\rho d}
$$

(iv) Finally, to obtain the electric field energy per unit length we must define $Q$ per unit length of the cylinder. In Eq. (40) we introduced $\sigma$, the density of the $\mathrm{U}(1)$ charge per unit area (in the units of $g^{2} / 2$ ). It is convenient then to introduce $Q$ as

$$
\sigma(2 \pi \rho)=Q \frac{g^{2}}{2} .
$$

The electric field energy per unit length takes the form

$$
\frac{1}{16 \pi} g^{2} Q^{2} \frac{d}{\rho} \text {. }
$$

As a result, the total tension of the $Q$ cylinder (per its unit length) is the following function of $\rho$ and $d$ :

$$
\begin{aligned}
& T_{Q-\text { cylinder }}(\rho, d)=\frac{g^{2} \xi^{2} \pi}{4} \rho d+\frac{4 \pi(\Delta m)^{2}}{g^{2}} \frac{\rho}{d}+\frac{4 \pi n^{2}}{g^{2}} \frac{1}{\rho d}+\frac{Q^{2} g^{2}}{16 \pi} \frac{d}{\rho} \text {. } \\
& \text { Coulomb phase Kinetic a Magnetic field Electric field }
\end{aligned}
$$

We do minimization in two steps. First we minimize the "Coulomb" and the magnetic field terms to obtain the product $\rho d$ at the minimum. Then we minimize the kinetic- $a$ and the electric field terms to obtain the ratio $\rho / d$ at the minimum. In this way we arrive at (the asterisk denotes the values at the minimum)

$$
(\rho d)_{*}=\frac{4 n}{g^{2} \xi}, \quad\left(\frac{\rho}{d}\right)_{*}=\frac{Q g^{2}}{8 \pi(\Delta m)} .
$$

Inserting these critical values in Eq. (58) we get the tension of the $Q$ cylinder,

$$
T_{Q-\text { cylinder }}=2 \pi \xi n+Q(\Delta m) .
$$

For consistency we must require $(\rho / d)_{*} \gg 1$. 


\subsection{Implementation of the $Q$ cylinder in the sigma- model limit}

It turns out that in the sigma-model limit an analog of the $Q$ cylinder was discovered and studied long ago, see Ref. [17] where it goes under the name of a $Q$-lump. We will reserve the name $Q$ lump for $(2+1)$-dimensional theory in which this soliton must be considered as a particle. In $(3+1)$-dimensional theory we are interested in Leese's soliton can be called $Q$ string. The $Q$ string is characterized by a topological (instanton) number $n$ and the global $\mathrm{U}(1)$ charge per unit length $Q$. In essence, it is a combination of the BelavinPolyakov instanton [22] in the two-dimensional $\mathrm{CP}(1)$ model with $Q$ charging. What remains to be done is to identify the instanton quantum number, the topological charge $n$, with the magnetic flux in the full theory (in our normalization the magnetic flux is $4 \pi n$ ). We will see shortly that this identification is correct. Thus, in the sigma-model limit the magnetic flux of the $Q$ cylinder becomes related to the homotopy group $\pi_{2}\left(S^{2}\right)$. In other words, it is related to the number of times the plane is mapped onto the target-space sphere. A special feature of the Leese-type $Q$ charged soliton is that the soliton solution approaches the vacuum asymptotics in a power-like rather than exponential manner.

The conventional Belavin-Polyakov instanton [22] (for a review see [10]) has two moduli: the size and the $\mathrm{U}(1)$ phase. The size is a modulus since the $\mathrm{CP}(1)$ model considered by Belavin and Polyakov is classically scale invariant.

Addition of the twisted mass in the sigma model and associated with it potential brakes the scale invariance. The instanton thus tends to shrink to zero size and become singular. A way to prevent this collapse is to $Q$ charge the soliton, i.e. to give a time-dependent rotation to the $\mathrm{U}(1)$ phase. Then the size modulus gets fixed in terms of $Q$.

To obtain the exact solution for the $Q$ string in the sigma model we perform the following Bogomol'nyi completion:

$$
\begin{aligned}
T_{Q-\text { string }} & =\xi \int d^{2} x\left[\left|\frac{\partial_{t} u \pm i(\Delta m) u}{1+\bar{u} u}\right|^{2}+\frac{1}{2}\left|\frac{\partial_{i} u \pm i \epsilon_{i j} \partial_{j} u}{1+\bar{u} u}\right|^{2}\right. \\
& \left. \pm i(\Delta m) \frac{\bar{u} \partial_{t} u-u \partial_{t} \bar{u}}{(1+\bar{u} u)^{2}} \mp \frac{i}{2} \epsilon_{i j} \frac{\partial_{i} \bar{u} \partial_{j} u-\partial_{j} \bar{u} \partial_{i} u}{(1+\bar{u} u)^{2}}\right]
\end{aligned}
$$


The $\mathrm{U}(1)$ charge per unit length is

$$
Q=\xi \int d^{2} x i \frac{\bar{u} \dot{u}-u \dot{\bar{u}}}{(1+\bar{u} u)^{2}},
$$

while the last term is proportional to the instanton invariant

$$
n=-\frac{i}{4 \pi} \epsilon_{i j} \frac{\partial_{i} \bar{u} \partial_{j} u-\partial_{j} \bar{u} \partial_{i} u}{(1+\bar{u} u)^{2}}
$$

For 1/2 BPS-saturated $Q$ string the first line in Eq. (61) must vanish. Choosing the lower signs the saturating solution can be written as

$$
u(z, t)=u_{0}(z) e^{i(\Delta m) t}
$$

where we introduce the complex variable $z=x_{1}-i x_{2}$. The equation for $u_{0}(z)$ is then the Belavin-Polyakov instanton equation (self-duality equation) which has a solution

$$
u_{0}(z)=\left(\frac{c}{z}\right)^{n}
$$

where $c$ is a constant, while the power $n$ coincides with the instanton number in Eq. (63). Calculating $Q$ for the solution (64), (65) we find

$$
Q=\xi(\Delta m)|c|^{2} \frac{2 \pi^{2}}{n^{2}}\left(\sin \frac{\pi}{n}\right)^{-1}
$$

For the minimal winding, $n=1$, the charge density $Q$ is infinite: in this case Eq. (62) is logarithmically divergent. This is due to the fact that the Leese soliton approaches the vacuum field in a power-like manner. Only at large $n$ it becomes reminiscent of a thin-wall cylinder. And even at large $n$ the relation with the worldvolume description of Sect. 4 is not straightforward. Indeed, in the worldvolume description the phase $\alpha$ could be any linear function of $t$, while for the $Q$ string at hand the phase is $(\Delta m) t$, with the unambiguously fixed coefficient. The charge density $Q$ can still be arbitrary since it quadratically depends on the instanton size $|c|$, see Eq. (66). If $Q$ is fixed, so is the $Q$ string transverse size. This is not a feature of the worldvolume description.

After these remarks we can finally give the expression for the $1 / 2$ BPSsaturated $Q$ string tension,

$$
T_{Q-\text { string }}=2 \pi \xi n+(\Delta m) Q \text {. }
$$


This formula confirms our interpretation of the instanton number $n$ as the magnetic flux in the microscopic theory.

In this model, thanks to the underlying supersymmetry, there is a considerable amount of exact analytic information for studying the important issue of $Q$ string stability. They are stable against decay into elementary quanta of the $u$ field perturbed around the vacuum.

The solution (65) is a limiting case of the $n$-instanton solution, which is known in the general case. A generic solution with the arbitrary instanton number $n$ can be written as

$$
u(z, t)=\left(\sum_{i=1}^{n} \frac{c_{i}}{z-z_{i}}\right) e^{i \Delta m t}
$$

where the moduli $c_{i}$ are complex numbers subject to the constraint

$$
\sum_{i}^{n} c_{i}=0
$$

This polygonal relation ensures finiteness of the $Q$ charge (per unit length)

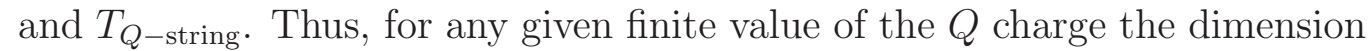
of the moduli space of the $Q$ string is $\nu=4 n-2$.

\subsection{Comparing the bulk-theory and sigma model ap- proximations}

It is instructive to compare results for the $Q$ cylinder obtained in various approximations. First, we observe with satisfaction that the expressions for the tension, Eqs. (60) and Eq. (67), coincide. It is instructive to make one step further. Let us discuss the moduli space of the $Q$ cylinder. The sigma model analysis demonstrates that the $Q$ string in the topological sector $n$ has a moduli space of solutions of dimension $4 n-2$. For our consideration to be valid we must assume $n \gg 1$. In this limit we deal with an infinitedimensional moduli space in the sigma-model description. Is there a trace of this phenomenon in the bulk-theory description?

Although, as was noted in Sect. 4.2, the relation between these two descriptions is not straightforward, one can think that the answer is positive. From the tension formula (58) we see that the same tension as for a "round cylinder" is obtained for a generic non-spherically symmetric $Q$ cylinder with 
the perimeter $2 \pi \rho_{*}$. Any closed curve with the fixed perimeter gives rise to an equally energetic $Q$ cylinder (see Fig. 9) as long as one can neglect terms depending on the curvature of the cylinder walls. This can be viewed as a remnant of the moduli space in the sigma-model description.
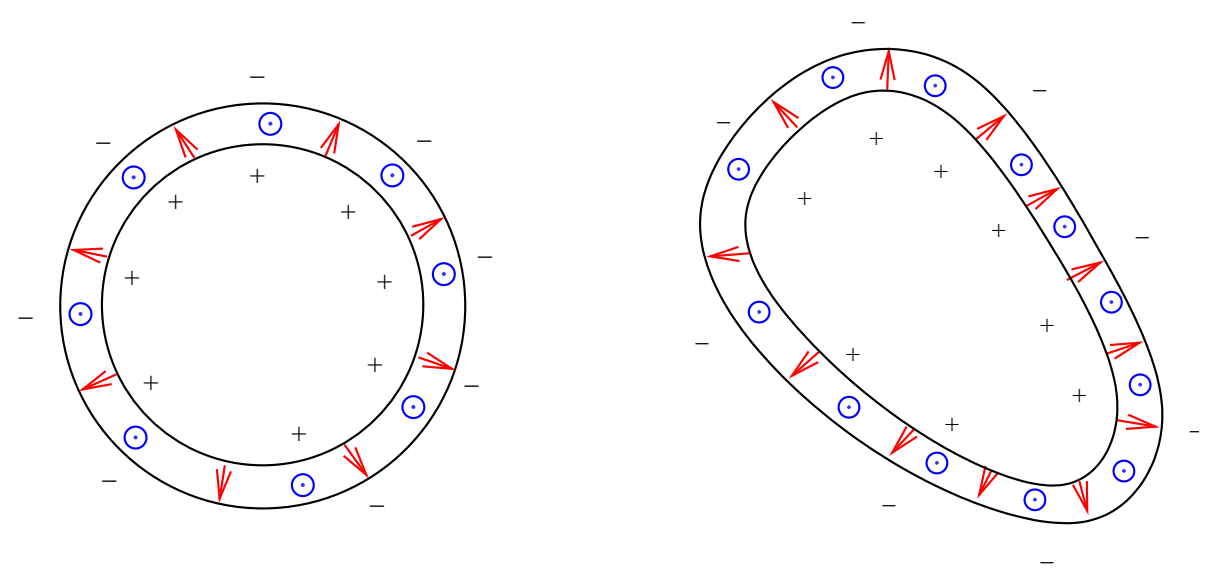

Figure 9: Moduli space of the $Q$ lump at large magnetic flux.

Now let us discuss the issue of a possible $Q$ charge leakage from the cylinder through the emission of elementary quanta $(Q$ stability). This discussion runs in parallel to that of the wall $Q$ stability, Sect. 3.3. The $Q$ cylinder tension (see Fig. 10) implies that the $Q$ cylinder is marginally stable under the $Q$ charge radiation via emission of mesons. Mesons, in the sigma model approximation, are the quanta of the $u$ field. They have mass $\Delta m$ and carry $Q=1$. In the full bulk theory the meson is a composite object composed of a quantum of the $q_{2}$ field screened by a $\bar{q}_{1}$. From Table 1 we see that this composite object has the vanishing electric charge and the $\mathrm{U}(1)$ charge $Q=1\left(\frac{1}{2}\right.$ from the $q_{2}$ and $\frac{1}{2}$ from $\left.\bar{q}_{1}\right)$.

Finally, let us discuss the fact that in the sigma model analysis the frequency $\omega$ is fixed, while it seems to be arbitrary in the worldvolume description.

We will show that in the bulk-theory description at the end of the day we also get this feature, a specific value of the frequency. To this end we can confront two expressions for the electric field obtained in different ways. On the one hand, it is quite trivial that

$$
E=\frac{Q g^{2}}{2} \frac{1}{2 \pi \rho}
$$




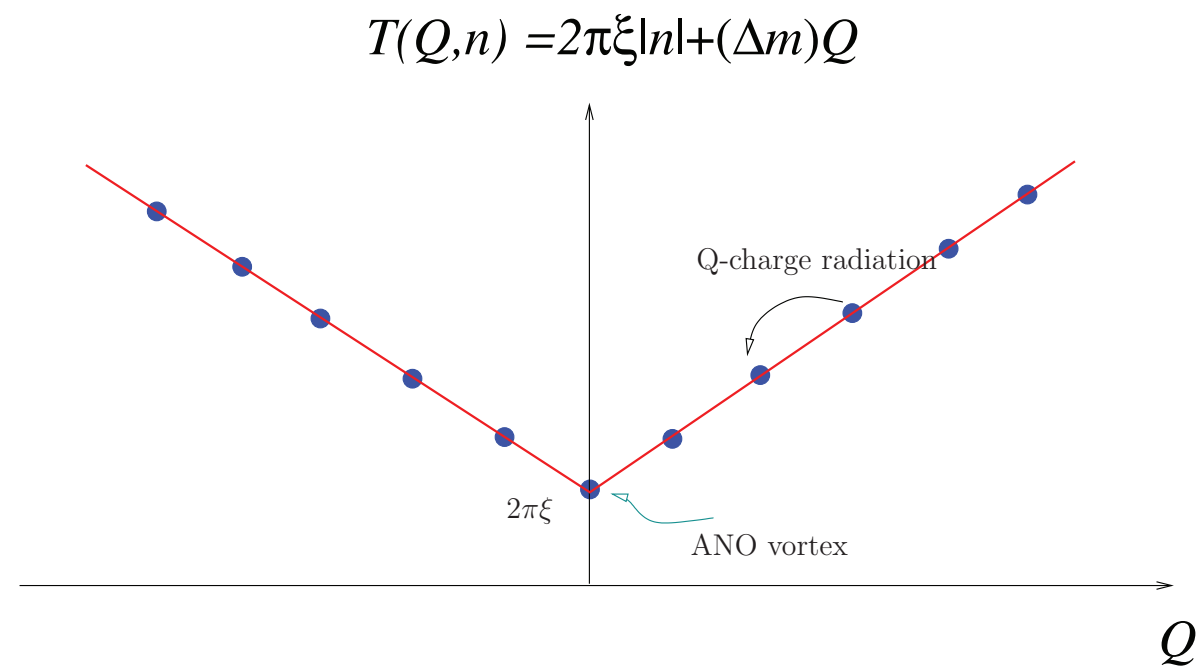

Figure 10: The $Q$ cylinder tension as function of $Q$ at fixed $n$. The $Q$ cylinder is marginally stable with respect to radiation of meson quanta with the $Q$ charge 1 .

On the other hand, from the value of the gauge potential in Table 3 we find

$$
E=\frac{2 \omega}{d} .
$$

Comparing these two expressions we get

$$
\frac{\rho}{d}=\frac{Q g^{2}}{8 \pi \omega},
$$

which in turn must be compared with Eq. (59). The result is consistent with (59) if $\omega=\Delta m$.

\subsection{Twisting the cylinder}

Now, we will make the next step in our program — we will "twist" the magnetic flux lines. The easiest way to explain the twist is through the worldvolume theory.

For an infinite flat wall the orientation of the magnetic field (which is in one-to-one correspondence with $\vec{\nabla} \alpha$ ) is unimportant since we can always align the $x$ axis with $\vec{\nabla} \alpha$. This ceases to be the case when we compactify the 


\begin{tabular}{|c|c|c|}
\hline & $\rho=0$ & $\rho \rightarrow \infty$ \\
\hline$q_{1}(\rho ; \theta, t)$ & 0 & $\sqrt{\xi} e^{-i \frac{\omega}{2}} t e^{i n \theta}$ \\
\hline$q_{2}(\rho ; t)$ & $\sqrt{\xi} e^{i \frac{\omega}{2} t}$ & 0 \\
\hline$\phi(\rho)$ & $-\sqrt{2} m_{1}$ & $-\sqrt{2} m_{2}$ \\
\hline$A_{\theta}(\rho)$ & 0 & $\frac{n}{\rho}$ \\
\hline$A_{t}(\rho)$ & $\omega$ & $-\omega$ \\
\hline
\end{tabular}

Table 3: Profile functions for the $Q$ string in the bulk theory

$x$ direction. Let us stick to the convention that the compactified direction is $x$. Typically, $\vec{\nabla} \alpha$ will be misaligned. What is the physical picture behind this misalignment?

It is quite obvious that compactifying the $x$ direction and choosing

$$
\alpha=a x+\tilde{a} y, \quad a \neq 0, \quad \tilde{a} \neq 0
$$

we twist the magnetic field inside the wall with regards to the orientation of the cylinder axis (Fig. 11). A similar situation was discussed in the second paper in Ref. [14]. The orientation of the magnetic field is given by the vector

$$
B_{i} \propto \varepsilon_{i j} a^{j}, \quad a^{j}=\{a, \tilde{a}\}
$$

Note that in this geometry the coefficient $a$ is quantized while $\tilde{a}$ is not. At the next stage, when the cylinder is folded to make a torus, $\tilde{a}$ will be quantized too.

One must $Q$ charge the twisted cylinder to make it stable just in the same vein as it was done in Sect. 4 (see Fig. 11).

\section{Twisted and $Q$ charged torus}

This section is central in the conceptual design of supersymmetric $Q$ solitons of arbitrary genus. Here we will explain how one can build a genus- 1 structure using the elements introduced above. To this end one glues the twisted cylinder of Sect. 4.4 to produce a torus (see Fig. 12). 


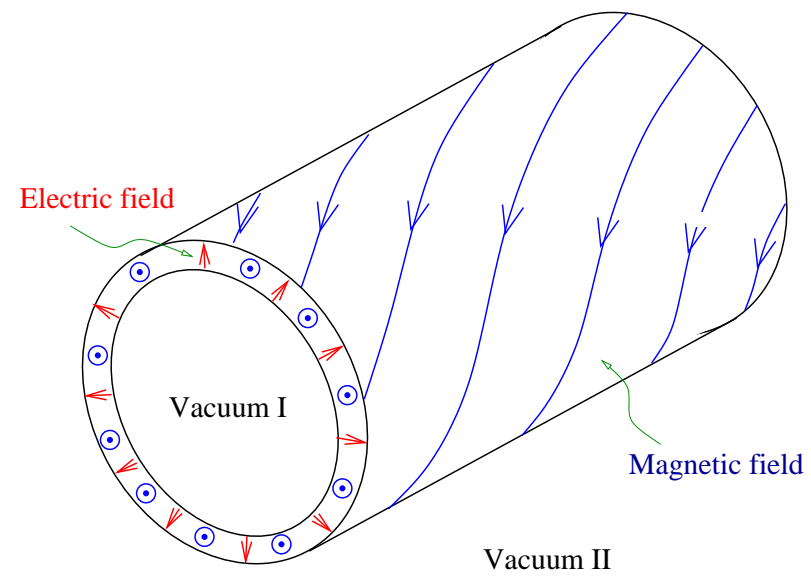

Figure 11: The flux line of the magnetic field inside the wall at $a \neq 0, \quad \tilde{a} \neq 0$.

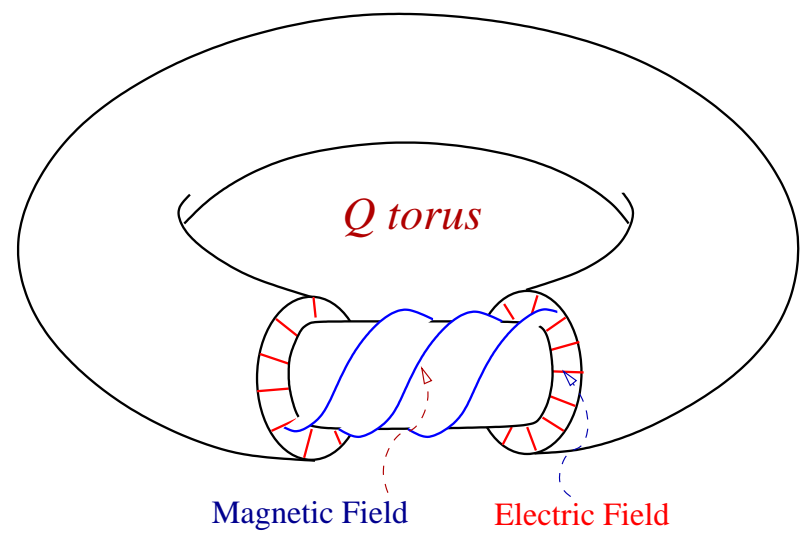

Figure 12: Twisted torus.

If both sizes of the fundamental cell $L_{x}, L_{y}$ are large (compared to the wall thickness) one can write

$$
\alpha=2 \pi k \frac{x}{L_{x}}+2 \pi \tilde{k} \frac{y}{L_{y}}
$$

where the integers $k$ and $\tilde{k}$ quantize $a$ and $\tilde{a}$. Correspondingly, the fluxes of the magnetic fields through both cycles of the torus get quantized too. The third quantum number needed to get a stable genus- 1 soliton is its $Q$ charge. 


\section{$5.1 \quad Q$ torus in the full bulk theory}

In the full bulk theory the $Q$ torus is a very complicate soliton; all fields of the bosonic Lagrangian (4) are exited. The only symmetry that we can use is the cylindrical one.

Thus, we introduce cylindrical coordinates: $r, z, \phi$. The profile functions will have non-trivial dependence on both $r$ and $z$, and, in some cases, also a phase rotation with regards to $\phi$ and $t$. The boundary conditions are imposed on two circles (see Fig. 13). One is the internal circle $z=0$ and $r=R$. The other is the external circle composed by the axial line $r=0$ plus the point at infinity. We summarize the profile functions and their boundary conditions in Table 4 .

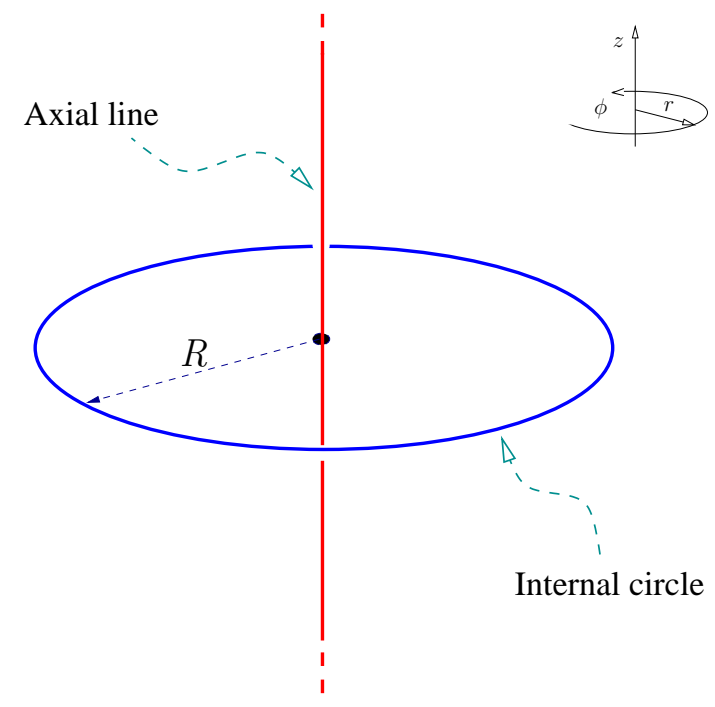

Figure 13: The boundary conditions for the $Q$ torus profile functions (see Table 4), are given on two circles. The first one is the internal circle (Vacuum $I$ ), the other circle is the axial line plus the point at infinity (Vacuum $I I$ ).

A comment is in order here to explain not yet defined quantities in Table 4 summarizing the boundary conditions. First of all, $R$ is the radius of the internal circle, also shown in Fig. 13. This parameter is not fixed; it is to be determined by energy minimization of the soliton. Another interesting quantity is the function $f_{n}(z)$ that appears in the boundary condition on the 


\begin{tabular}{|c|c|c|}
\hline & Internal circle & Axial line \\
\hline$q_{1}(r, z ; \phi, t)$ & 0 & $\sqrt{\xi} e^{-i \frac{\omega}{2} t} f_{n}(z)$ \\
\hline$q_{2}(r, z ; \phi, t)$ & $\sqrt{\xi} e^{i \frac{\omega}{2} t} e^{i N \phi}$ & 0 \\
\hline$a(r, z)$ & $-\sqrt{2} m_{1}$ & $-\sqrt{2} m_{2}$ \\
\hline$A_{\phi}(r, z)$ & $N / r$ & 0 \\
\hline$A_{r}(r, z)$ & 0 & 0 \\
\hline$A_{z}(r, z)$ & 0 & $-i f_{n}^{\prime}(z) / f_{n}(z)$ \\
\hline$A_{t}(r, z)$ & $\omega$ & $-\omega$ \\
\hline
\end{tabular}

Table 4: Profile functions for the $Q$ torus in the bulk theory.

axial line for the scalar field $q_{1}$. This field must wind $n$ times around the vacuum manifold $S^{1}$ when one passes from $-\infty$ to $+\infty$ on the axial line. It is just a one-dimensional texture whose profile function $f_{n}(z)$ is not determined a priori and must be derived from energy minimization. The $Q$ torus is thus quite a complicated solitonic object: it has another "small" soliton in its boundary conditions!

Needless to say, this object is quite resilient to direct solution even using numerical methods since a set of entangled partial differential equations is involved. The main purpose of this paper is to prove the existence of an appropriate soliton and its stability. To this end we will use the two approximations we exploited above to describe the $Q$ wall and $Q$ cylinder. Let us start from the bulk-theory description. In vein with similar assumptions for the $Q$ wall and $Q$ cylinder, we will impose the following constraint on the parameters of the $Q$ torus:

$$
R \gg \rho \gg d \gg \delta_{\text {edge }}
$$

As usual, $\delta_{\text {edge }}$ is set to zero; thus the $Q$ torus depends on three parameters: the total $\mathrm{U}(1)$ charge $q$, and two magnetic fluxes $n$ and $N$. In the particular limit (74) we can write the mass of the $Q$ torus as a function of three 
geometric parameters $R, \rho$ and $d$,

$$
\begin{aligned}
M(R, \rho, d)= & \frac{\pi^{2} g^{2} \xi^{2}}{2} R \rho d+\frac{8 \pi^{2}(\Delta m)^{2}}{g^{2}} \frac{R \rho}{d} \\
\text { Coulomb phase } \quad \text { Kinetic a } & \\
+ & \frac{8 \pi^{2} n^{2}}{g^{2}} \frac{R}{\rho d}+\frac{8 \pi^{2} N^{2}}{g^{2}} \frac{\rho}{R d}+\frac{q^{2} g^{2}}{32 \pi^{2}} \frac{d}{R \rho}, \\
& \text { Magnetic flux } n \quad \text { Magnetic flux } N \quad \text { Electric field }
\end{aligned}
$$

where we introduced the total charge (measured in integers)

$$
2 \pi R Q=q
$$

The first and the second terms in Eq. (75) are obtained from those in Eq. (58) by multiplying by $2 \pi R$. The third and the fourth terms represent the third term in Eq. (58) which splits into two terms due to the fact that there are two magnetic cycles now. The last term in Eq. (75) corresponds to the last term in Eq. (58).

To minimize this function we rewrite it in the following way:

$$
\begin{aligned}
M(R, \rho, d) & =\frac{\pi^{2} g^{2} \xi^{2} R}{2} \rho d+\frac{8 \pi^{2} n^{2} R}{g^{2}} \frac{1}{\rho d}+ \\
& +\frac{8 \pi^{2}(\Delta m)^{2} R}{g^{2}} \frac{\rho}{d}+\frac{8 \pi^{2} N^{2}}{g^{2} R} \frac{\rho}{d}+\frac{q^{2} g^{2}}{32 \pi^{2} R} \frac{d}{\rho} .
\end{aligned}
$$

Now, the first line can be minimized with respect to $\rho d$ while the second line can be minimized with respect to $d / \rho$, keeping $R$ fixed. In this way we arrive at

$$
(\rho d)_{*}=\frac{4 n}{g^{2} \xi}, \quad\left(\frac{d}{\rho}\right)_{*}=\frac{16 \pi^{2}}{q g^{2}} \sqrt{(\Delta m)^{2} R^{2}+N^{2}},
$$

where the asterisk marks the optimized $Q$-torus values. Inserting these expressions back in Eq. (77) we get

$$
M(R)=4 \pi^{2} \xi n R+\frac{q}{R} \sqrt{(\Delta m)^{2} R^{2}+N^{2}} .
$$

Finally we can minimize $M(R)$ with respect to $R$ to obtain $M_{Q \text {-torus }}$. For arbitrary $N$ the function will be rather complicated. In the limiting case 


$$
(\Delta m) R \ll N
$$

the formula for $M_{Q \text {-torus }}$ is simple, however. Indeed, minimizing

$$
M(R)=4 \pi^{2} \xi n R+\frac{q N}{R}
$$

we find

$$
R_{Q-\text { torus }}=\frac{1}{2 \pi \sqrt{\xi}} \sqrt{\frac{q N}{n}}, \quad M_{Q-\text { torus }}=4 \pi \sqrt{\xi} \sqrt{q N n} .
$$

Note that all the conditions in Eq. (74) as well as $(\Delta m) R \ll N$, can be satisfied under an appropriate choice of the charge $q$ and the magnetic fluxes $n, N$. Equation (78) implies

$$
\rho_{*}=\frac{1}{2 \pi \sqrt{\xi}} \sqrt{\frac{n q}{N}}, \quad d_{*}=\frac{8 \pi}{g^{2} \sqrt{\xi}} \sqrt{\frac{n N}{q}} .
$$

The conditions (74) now become

$$
\frac{1}{2 \pi \sqrt{\xi}} \sqrt{\frac{q N}{n}} \gg \frac{1}{2 \pi \sqrt{\xi}} \sqrt{\frac{n q}{N}} \gg \frac{8 \pi}{g^{2} \sqrt{\xi}} \sqrt{\frac{n N}{q}} \gg \frac{1}{g \sqrt{\xi}} .
$$

Here $(g \sqrt{\xi})^{-1}$ is the edge thickness, to be considered as the smallest size in the problem at hand.

Multiplying by $\sqrt{\xi q N n}$ we obtain an equivalent constraint,

$$
\frac{1}{2 \pi} q N \gg \frac{1}{2 \pi} n q \gg \frac{8 \pi}{g^{2}} n N \gg \frac{1}{g} \sqrt{q N n} \text {. }
$$

The condition $(\Delta m) R \ll N$ implies

$$
\sqrt{\frac{N n}{q}} \gg \frac{\Delta m}{2 \pi \sqrt{\xi}}
$$

We remind that in the thin wall approximation $\Delta m / \sqrt{\xi} \gg 1$. It is not difficult to see that Eqs. (85) and (86) can always be satisfied with an appropriate choice of the integer parameters

$$
q \gg N \gg n, \quad n N \gg q
$$


The second of these two conditions is irrelevant since the constraint (86) is stronger.

To conclude this section let us give our final result (in the thin edge approximation). The $Q$ torus mass in this limit takes the form

$$
M_{Q-\text { torus }}=4 \pi \sqrt{\xi} \sqrt{q N n}\left(1+\mathcal{O}\left(\frac{\Delta m}{\sqrt{\xi}} \sqrt{\frac{q}{N n}}\right)\right) .
$$

On the other hand, in the limit opposite to (86),

$$
\sqrt{\frac{N n}{q}} \ll \frac{\Delta m}{2 \pi \sqrt{\xi}}
$$

when $q$ is very large, Eq. (79) implies that

$$
\frac{q(\Delta m)}{n \xi} \gg R_{*} \gg \frac{N}{\Delta m},
$$

and

$$
M_{Q-\text { torus }}=q(\Delta m) .
$$

\section{2 $Q$ torus in the sigma-model limit}

Now let us look at the $Q$ torus soliton from a different perspective, that of the sigma-model limit (i.e. $\Delta m \ll g \sqrt{\xi}$ ). It turns out that in this limit $Q$ torus is related, in a way, to the Hopf Skyrmions discovered in $[23,24]$ (for a recent work see [25]).

In the sigma-model approximation the $Q$ torus is a non-trivial element of the Hopf homotopy group

$$
\pi_{3}\left(\mathbf{S}^{2}\right)=\mathbf{Z}
$$

An illustration that may help one to understand geometry of the Hopf map is given in Fig. 14.

Let us imagine the space $\mathbf{R}^{3}$ as a "book." Every "page" of the book is a semi-infinite plane attached to the axial line. The axial line plus the points at infinity are mapped onto the north pole of the target space $\mathbf{S}^{2}$. The pre-image of the south pole is a circle linked with the axial line. Every semi-infinite plane is wrapped around the target space. 


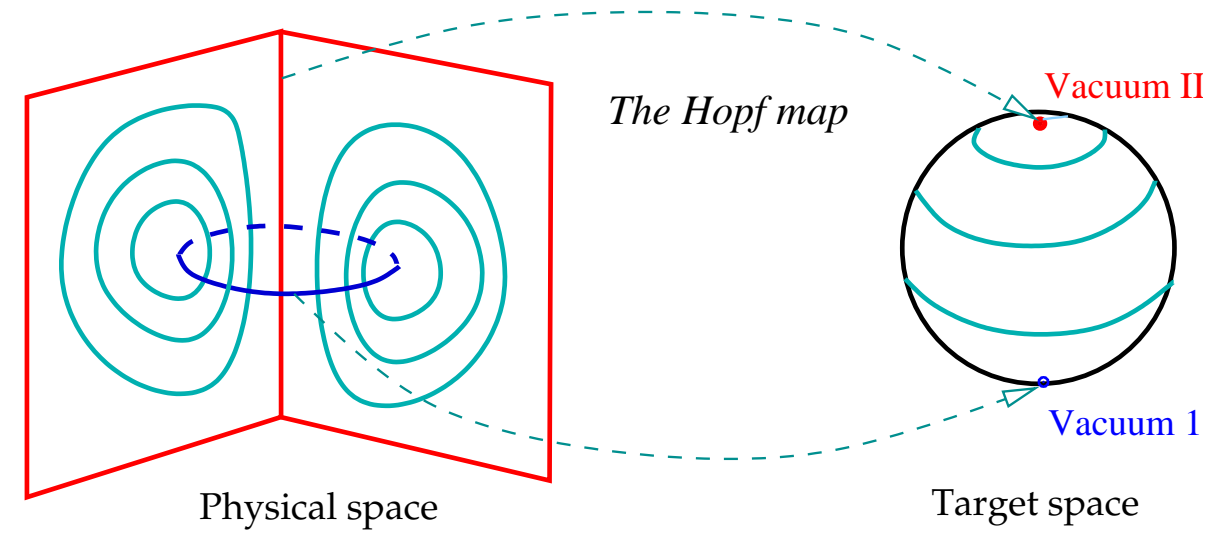

Figure 14: The Hopf map from the space $\mathbf{R}^{2}$ to the target space $\mathbf{S}^{2}$. The axial line plus the points at infinity are mapped onto the north pole (Vacuum $I I$ ). The internal circle is mapped onto the south pole (Vacuum $I$ ).

The U(1) phase - in Fig. 14 it is the rotation of $\mathbf{S}^{2}$ which keeps fixed the north and the south poles - is twisted as the semi-infinite plane is rotated around the axial line.

A comment is in order here to explain the stability of this Hopf Skyrmion. In the theory described by the Lagrangian (14), the Derrick theorem forbids [26] the existence of particle-like solitons. Even if the homotopy group creates nontrivial maps from the coordinate space to the target space, the kinetic and the potential terms for the scalar field cannot create a repulsive force necessary in order to prevent the soliton from collapsing. A way out suggested in $[23,24]$ was adding higher derivative terms. To avoid the Derrick collapse without introducing higher derivative terms in the Lagrangian we exploit a linear-in-time rotation of the $\mathrm{U}(1)$ phase which stabilizes the soliton through a centrifugal force.

\subsection{Comment on the cycles' stability}

A remark is in order here to better explain the stability of the cycles relevant to the twisted torus. This remark will help us avoid a possible source of confusion.

Consider the $Q$ torus in Fig. 15. The two non-trivial cycles we have are denoted by $A$ and $B$ in this figure. The $A$ cycle has radius $\rho$ while the $B$ cycle has radius $R$. The magnetic fluxes are $4 \pi n$ (the one that goes parallel 
to the $B$ cycle) and $4 \pi N$ (the one that goes parallel to the $A$ cycle). A crucial point we want to stress is that the magnetic flux stabilizes the cycle that crosses it rather than the cycle which runs parallel to it.

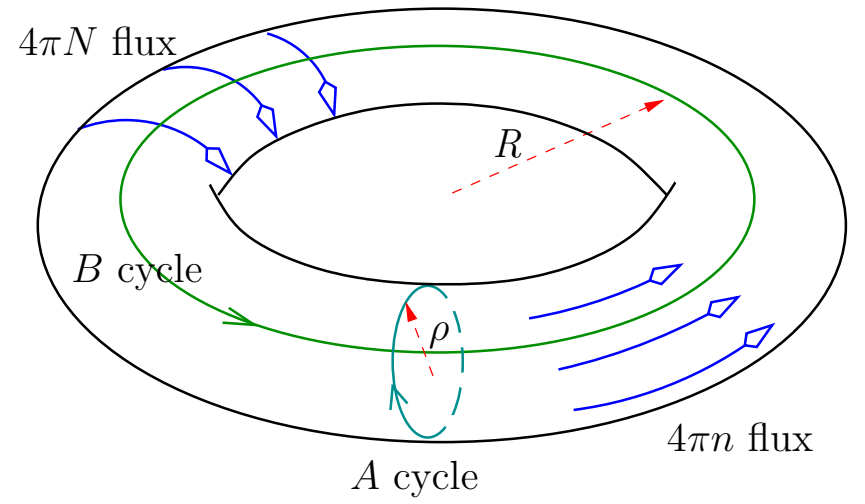

Figure 15: Two nontrivial homology cycles associated with the $Q$ torus: the $A$ cycle with radius $\rho$ and the $B$ cycle with radius $R$. Note that the $A$ cycle is stabilized by the $4 \pi n$ magnetic flux that passes along the $B$ cycle. And vice versa, the $B$ cycle is stabilized by the $4 \pi N$ magnetic flux that goes along the $A$ cycle.

For example, the flux $4 \pi n$ is responsible for the stability of the $A$ cycle and vice versa. In Sect. 5.1 we obtained quite simple formulae for the mass and dimensions of the $Q$ torus. We can use these expressions to better understand the issue of stability. First of all, let us note that $M_{Q \text {-torus }}$ is proportional to $\sqrt{q n N}$ which means that all three integer parameters, $q, n$ and $N$, are crucial for the existence of the $Q$ torus. If one of them vanishes the $Q$ torus disappears.

The radius $R$ is proportional to $\sqrt{q N / n}$ and we see that its stability is guaranteed, in fact, by the electric field $q$ and the magnetic flux $N$. The radius $\rho$ is proportional to $\sqrt{q n / N}$ implying that its stability is guaranteed by $q$ and the magnetic flux $n$. The roles of two magnetic fluxes are complementary.

\subsection{Interactions between $Q$ tori}

In this section we will discuss the issue of interaction between $Q$ tori. This process is important if we want to establish a condition for the existence of higher-genus solitons. At the end of this section we will be able to formulate this condition. 
First of all let us visualize possible physical processes which are allowed given conservation of fluxes and the $\mathrm{U}(1)$ charge. Let us consider the physical process of merging of $Q$ tori, that is two $Q$ tori fusing to become a single one. Equivalently, if viewed in the opposite time direction, this process describes a $Q$ torus decay into two smaller ones. For simplicity we will concentrate only on the merging direction.

Two alternatives are possible depending on whether the cycle involved is the $A$ cycle or the $B$ cycle (see Fig. 15 for our conventions regarding the cycle names).

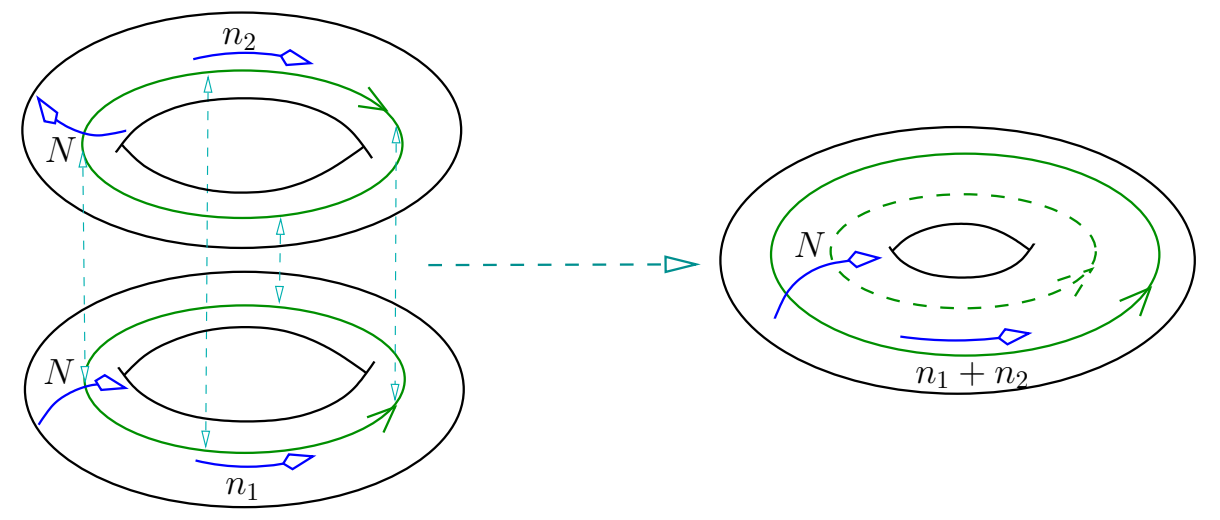

Figure 16: A "landing of two Flying Saucers", or merging of two $Q$ tori through their $A$ cycles. This process happens "vertically." The two $Q$ tori must have the same magnetic flux $N$; they touch on their $B$ cycles and then merge. We will refer to $A$ merging or vertical merging. Viewed in the opposite direction it is an $A$ or vertical decay.

To begin with, let us deal with the $A$ cycle merging (it is simpler). It is displayed in Fig. 16 and corresponds to the following process:

$$
\left(n_{1}, N, q_{1}\right)+\left(n_{2}, N, q_{2}\right) \longrightarrow\left(n_{1}+n_{2}, N, q_{1}+q_{2}\right) .
$$

The $A$ process (it can be called "vertical merging" as is clearly seen from Fig. (16), is possible only if two fusing $Q$ tori share the same $N$ flux.

The second process which we call $B$ merging (or horizontal merging) corresponds to merging of two $B$ cycles of two merging $Q$ tori. The corresponding reaction is

$$
\left(n, N_{1}, q_{1}\right)+\left(n, N_{2}, q_{2}\right) \longrightarrow\left(n, N_{1}+N_{2}, q_{1}+q_{2}\right),
$$


see Fig. 17. As is perfectly clear from Fig. 17 this process is more convoluted than the vertical one. It is possible only if two merging $Q$ tori share the same $n$ flux.

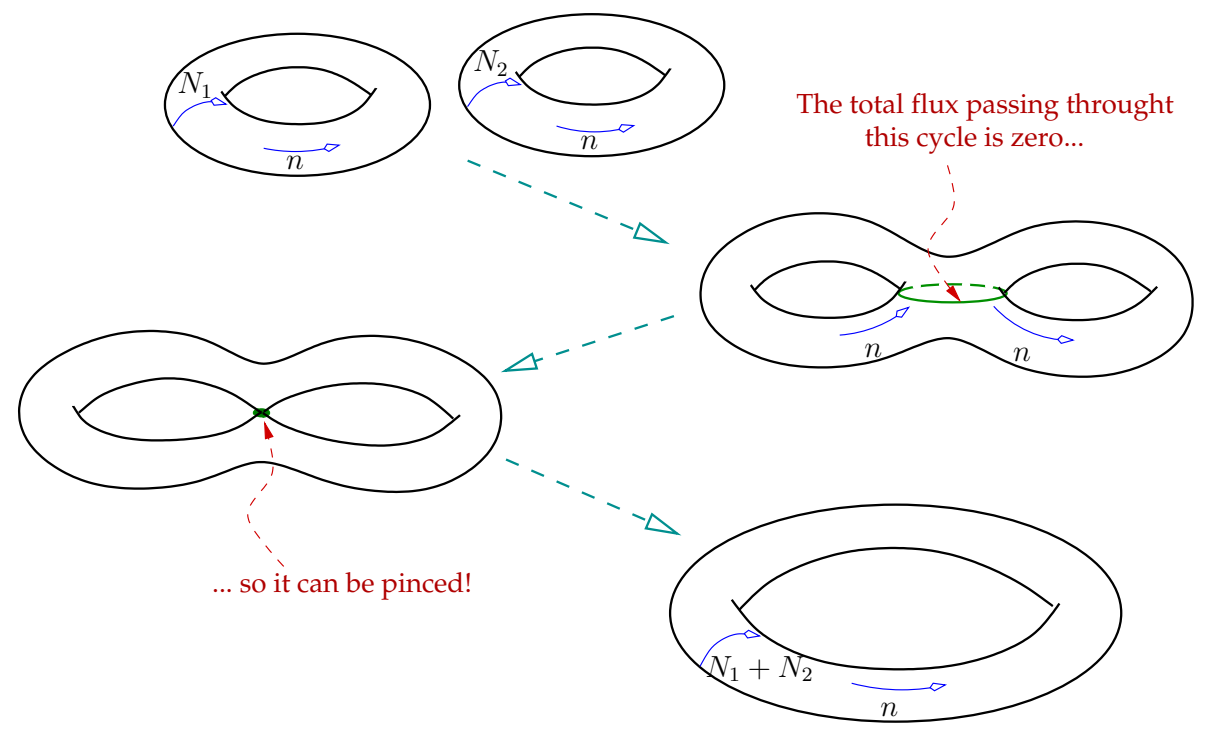

Figure 17: A "crush of two Flying Saucers" or merging of two $Q$ tori through their $B$ cycles. This process happens horizontally. The two merging $Q$ tori must have the same magnetic flux $n$. They touches and form a genus-2 $Q$ soliton. In fact, this is not a genuinely genus- 2 soliton: the middle handle has vanishing total magnetic flux passing trough it. Therefore, this handle is unstable and can be pinched away. What is left is a $Q$ torus of genus 1 . We can call it $B$ or horizontal merging. In the opposite direction it is a $B$ or horizontal decay.

The conditions imposed by the flux and charge conservation is not the end of the story. We must discuss the energy balance. This is a more complicated issue since it involves knowledge of the mass function $M_{Q-\text { torus }}$ which, so far, we have only in a certain limit. But we can at least speculate on the marginal stability.

For generic solitons which depend on a single integer, say, $n$, the mass is said to be marginally stable if it is a linear function of $n$. There is only one process of merging of such solitons, namely, an $n_{1}$ soliton adds to an $n_{2}$ soliton to form a compound $n_{1}+n_{2}$ soliton. Marginal stability with respect to this process constrains the mass function to be a linear function of $n$. 
In the case under discussion, the $Q$ torus, the soliton mass depends on three integers, and two processes are allowed. The marginal stability condition does constrain the mass function but still leaves a lot of freedom.

Note that minimization of (79) gives a function which is marginally stable (see e.g. (88)). We can easily prove this assertion by considering only the relevant parts of the formula (79),

$$
M(R)=n R+\frac{q}{R} \sqrt{R^{2}+N^{2}},
$$

where for simplicity we set all irrelevant parameters to 1 . Under the "vertical" rescaling

$$
(n, N, q) \rightarrow(a n, N, a q)
$$

we have to make the simultaneous rescaling

$$
M \rightarrow a M \text { and } R \rightarrow R .
$$

Under the "horizontal" rescaling

$$
(n, N, q) \rightarrow(n, b N, b q)
$$

we have to make the simultaneous rescaling

$$
M \rightarrow b M \text { and } R \rightarrow b R \text {. }
$$

The geometric meaning of these rescalings is represented in Fig. 18,

\subsection{Higher genera}

Now we can present a condition for a genus- $2 Q$ soliton to be a genuine genus-2 object and not something that could decay into a genus-1 $Q$ torus. In Fig. 19 we have depicted a $Q$ soliton with its nontrivial cycles and fluxes. The soliton now depends on five charges: the electric charge $q$, the magnetic fluxes $n_{1}$ and $n_{2}$ which stabilize the $A$ cycles and the magnetic fluxes $N_{1}$ and $N_{2}$ which stabilize the $B$ cycles. The soliton is genuinely genus- 2 if the two processes (91) and (92) are forbidden, namely

$$
n_{1} \neq n_{2}, \quad \text { and } \quad N_{1} \neq N_{2} .
$$

For higher genera the condition is more complicated, not just a straightforward generalization of (93). For example, take a genus-3 soliton with the 


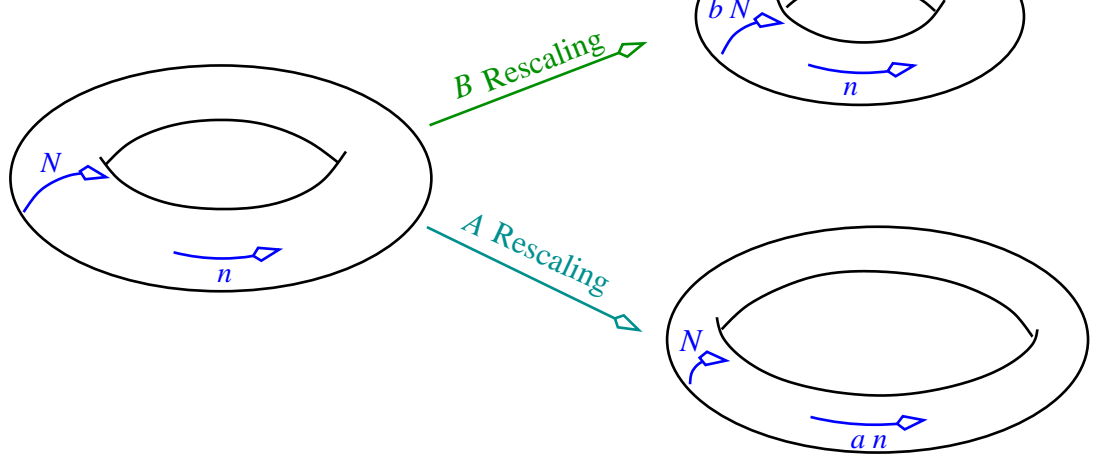

Figure 18: The $A$ and the $B$ rescalings.

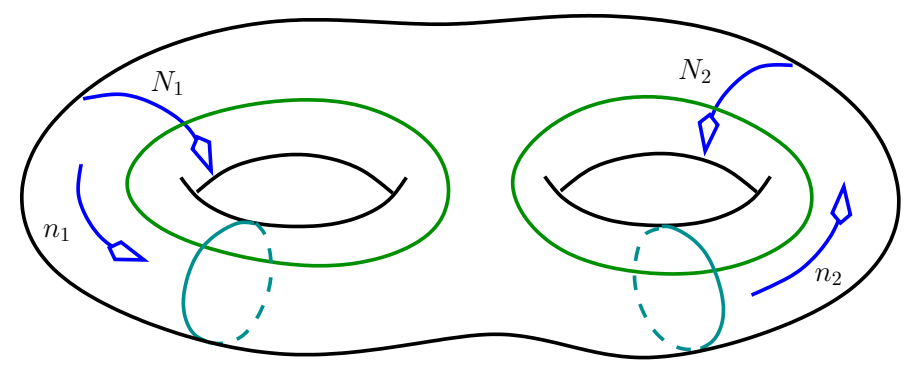

Figure 19: A $Q$ soliton of genus 2 .

fluxes $(1,1),(2,2)$ and $(3,3)$. At first sight, it might seem a genuine genus-3 object since none of the integers $n$ are equal and so are $N$ 's (they are unequal too). This implies that it cannot be reduced to lower-genus solitons through only one transition.

However, we must consider a possibility of multiple combined transitions. For instance, the last handle can split with by vertical decay into $(1,3)$ plus $(2,3)$. Then we will have four handles $(1,1),(1,3),(2,3)$ and $(2,2)$. The first two can now horizontally merge into $(1,4)$ while the last two can horizontally merge into $(2,5)$. An apparent genus-3 soliton thus turned out to be a genus2 soliton. The mathematical condition for genuine higher-genera solitons taking into account these multiple transition of combined decays/mergings is yet to be found. 


\subsection{Q charge radiation revisited}

The leakage of the $\mathrm{U}(1)$ charge through radiation of elementary $Q$ charged quanta was discussed more than once above. Here we would like to return to this question in connection with $Q$ stability of the $Q$ torus. This is a dynamical question and its answer crucially depends on the still unknown function $M(n, N, q)$. To find out whether or not a generic $Q$ torus $(n, N, q)$ is stable under the decay into a $Q$ torus $(n, N, q-1)$ plus a "meson" we have to confront the mass difference $M(n, N, q)-M(n, N, q-1)$ with the meson mass.

To analyze a concrete example let us take the mass formula (79) which in the case $\Delta m R \ll N$ leads to Eq. (82) for $M_{Q-\text { torus. From the square root }}$ $q$ dependence of $M_{Q \text {-torus }}$ we deduce that there is a critical value

$$
q_{*} \sim \sqrt[3]{\frac{\xi n N}{(\Delta m)^{2}}}
$$

below which the $Q$ torus is instable under the $Q$ charge radiation. Above $q_{*}$ the $Q$ torus is stable. This is not the end of the story, however.

To analyze the problem of $Q$ stability for arbitrary large values of $q$ we have to return to the mass formula (79) since the approximation $(\Delta m) R \ll N$ will certainly break down at

$$
q \sim \frac{\xi n N}{(\Delta m)^{2}}
$$

At $q \rightarrow \infty$ we can neglect the first term in Eq. (79) and directly minimize the second term. The minimum $M=q(\Delta m)$ is achieved at very large $R$ $(R \rightarrow \infty)$. This mass function, see Eq. (89)), is (asymptotically) tangential to the line of marginal stability. In Fig. 20 we sketch a qualitative behavior of the $Q$-torus mass as a function of $q$.

\section{The Dirac-Born-Infeld Action}

In Sects. 3, 4, and 5 we used the worldvolume theory framework to consider $Q$ walls, $Q$ cylinders and $Q$ tori. The effective $(2+1)$-dimensional action (25) describes low-energy dynamics of two massless moduli on the worldvolume of the domain wall, namely the translational fluctuation $z_{0}$ and the U(1) phase 


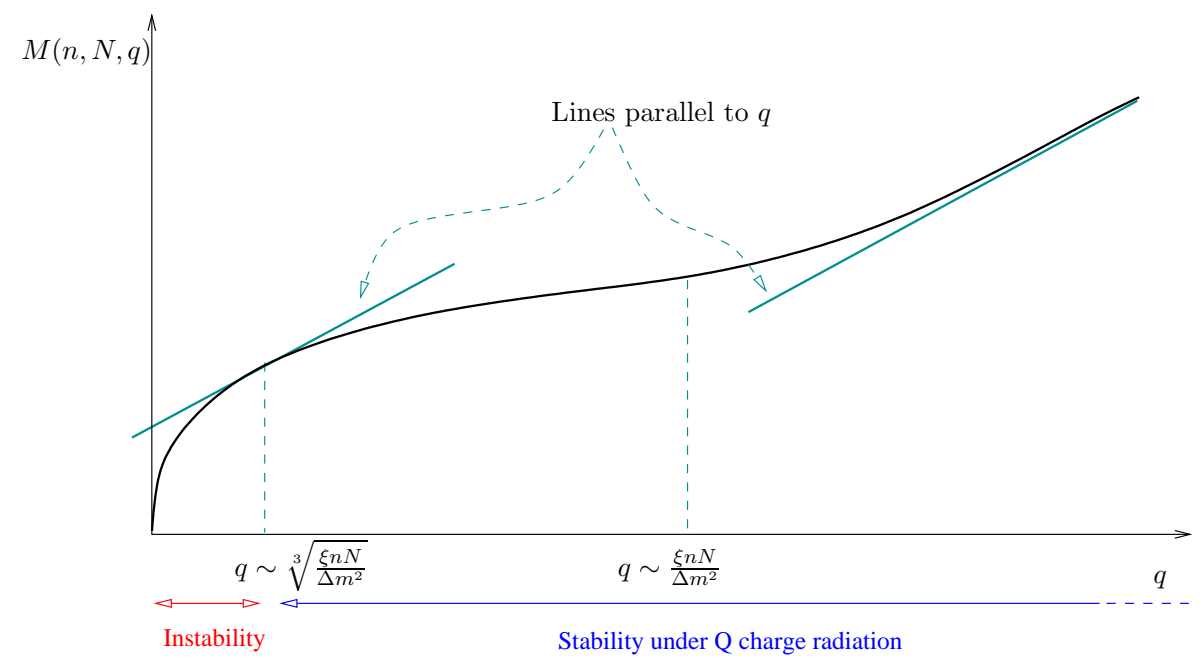

Figure 20: A schematic representation of $M_{Q-\text { torus }}$ vs. $q$ at fixed $n$ and $N$.

$\alpha$. Alternatively, one can deal with $(2+1)$-dimensional $\mathcal{N}=2 \quad \operatorname{SQED}(27)$. The expressions for the action we used in these sections were derived in [1]; they are valid to the lowest order in derivatives (quadratic in derivatives). Quartic and higher order derivative terms are not included in Eq. (27).

Needless to say, the full worldvolume action contains higher-order derivative terms. For weak magnetic and electric fields, in the low-energy limit, higher order-terms can be neglected. Then we have a free field theory (25) containing only the kinetic terms. However, higher-order terms are crucial (even in the low-energy limit) if we want to describe solutions with nontrivial geometry, such as the $Q$ string or $Q$ torus. After minimization, at the optimal values of $R$ and $d$, the magnetic and electric fields stabilizing the solitons need not be small.

The free theory (25) can describe weakly charged $Q$ walls, as we saw in Sects. 3.1] and 3.2, with crossed electric and magnetic fields, but it cannot fully reflect nontrivial geometry, say, of the $Q$ torus.

In order to obtain the effective worldvolume action including higher-order derivative terms we can rely on two very well-known principles: geometry and supersymmetry. Geometry tells us that the action on the brane must contain a term proportional to the volume of the domain wall. If we call $x^{\alpha}=(t, x, y)$ the coordinates on the wall worldvolume and $X^{\mu}=(T, X, Y, Z)$ 
the coordinates of space-time 4 the action must contain the term

$$
-\int d^{3} x \sqrt{-\operatorname{det} g_{\gamma \beta}}
$$

where $g_{\alpha \beta}$ is the pull-back of the space-time metric on the worldvolume.

Fixing the gauge of the local coordinates we can write the metric as

$$
g_{\alpha \beta}=\eta_{\gamma \beta}+\partial_{\gamma} z \partial_{\beta} z
$$

where $z$ is the translational modulus fluctuation (for brevity we omit the subscript 0). As we know, $z$ is the superpartner of the $\mathrm{U}(1)$ modulus $\alpha$; therefore, requiring supersymmetry we arrive at

$$
S=-T_{\mathrm{w}} \int d^{3} x \sqrt{-\operatorname{det}\left[g_{\gamma \beta}+\frac{1}{(\Delta m)^{2}} \partial_{\gamma} \alpha \partial_{\beta} \alpha\right]} .
$$

Dualizing the U(1) phase according to Eq. (23) we arrive at the Dirac-BornInfeld (DBI) action

$$
S=-T_{\mathrm{w}} \int d^{3} x \sqrt{-\operatorname{det}\left[g_{\alpha \beta}+\frac{1}{2 \pi \xi} F_{\alpha \beta}\right]} .
$$

If we expand the DBI action in the fields we get to the leading order

$$
S=\int d^{3} x\left[-T_{\mathrm{w}}-\frac{T_{\mathrm{w}}}{2} \partial_{\gamma} z \partial^{\gamma} z-\frac{1}{4 e^{2}} F_{\gamma \beta} F^{\gamma \beta}+\ldots\right],
$$

which exactly coincides with Eq. (25).

It is instructive to note that the coefficients in Eq. (99) automatically match standard expressions of string theory. The standard relative coefficient between $F_{\gamma \beta}$ and $g_{\gamma \beta}$ is $2 \pi \alpha^{\prime}$ where $\alpha^{\prime}$ is the slope of the Regge trajectory expressible in terms of the string tension as

$$
T_{\text {string }}=\frac{1}{2 \pi \alpha^{\prime}} \text {. }
$$

(For an introductory presentation see e.g. [27].) Therefore, approaching the DBI action from this side we we would expect the relative coefficient $1 / T_{\text {string }}$.

\footnotetext{
${ }^{4}$ In this section we adopt the metric conventions $\eta_{\gamma \beta}=(-1,1,1)$ for three-dimensional theory and $\eta_{\mu \nu}=(-1,1,1,1)$ for four-dimensional theory.
} 
That's exactly what we have in Eq. (99) since the tension of the ANO string in the problem at hand is $2 \pi \xi$.

At the moment it is not clear in which sense the DBI action is complete and adequate in the problem at hand. Certainly, it ceases to present adequate description at a certain energy scale where heavy degrees of freedom, either on the brane or in the bulk, must be taken into account. This assertion is true both in string theory, where we deal with the D2 brane of Type IIA superstring, and in our model where the brane is a domain wall of $\mathcal{N}=2$ SQED.

Another important point to be noted here is that the DBI action has a maximal value of the electric field. While the physical meaning of this is well understood in string theory, at the moment it is not quite clear in the field-theoretic context. We take it as a signal that the worldvolume description in terms of the moduli fields fails and other degrees of freedom become important. It could be related to the creation of a "vortex handle" on the worldvolume. This effect, essentially classical from the bulk point of view, is a quantum polarization of the vacuum from the $(2+1)$-dimensional standpoint.

The DBI action is known to have interesting solutions where the crossed electric and magnetic fields affect global geometry of the brane. One example is the supertube solution [28]. The supertube is a D2 brane with cylindrical geometry. It is a bound state of F1 strings and D0 branes which in the large-flux limit becomes well described by the cylindrical D2-brane. In the field-theoretic context it corresponds to the $Q$ string [8] (see our discussion in Sect. 4.2). Feld-theoretic implementations of the supertube solution in other (albeit related) models are discussed in [29].

Now we will show that the DBI action admits a solution with toric geometry which corresponds to the $Q$ torus we discussed at length in the previous sections.

\subsection{Construction of the $Q$ torus from the DBI action}

Let us parametrize the worldvolume coordinates as $x^{\alpha}=(t, \theta, \phi)$ where $\theta, \phi$ are two angles defined on the interval $[0,2 \pi]$. The space-time is parametrized by cylindrical coordinates $X^{\mu}=(T, Z, R, \Phi)$. 
We fix the worldvolume reparametrization invariance in the following way:

$$
\begin{aligned}
& T=t, \quad \Phi=\phi ; \\
& R(\theta+2 \pi)=R(\theta) ; \\
& Z(\theta+2 \pi)=Z(\theta) .
\end{aligned}
$$

The only reparametrization freedom left is $\theta \rightarrow f(\theta)$. The induced metric on the worldvolume is

$$
\partial_{\gamma} X^{\mu} \partial_{\beta} X_{\mu}=\left(\begin{array}{ccc}
-1 & 0 & 0 \\
0 & R^{\prime 2}+Z^{\prime 2} & 0 \\
0 & 0 & R^{2}
\end{array}\right) .
$$

The field strength is

$$
F_{\gamma \beta}=\left(\begin{array}{ccc}
0 & E_{\theta} & E_{\phi} \\
-E_{\theta} & 0 & B \\
-E_{\phi} & -B & 0
\end{array}\right)
$$

Remember that now we deal with the dual formulation of the bulk theory: the magnetic field corresponds to the electric field in bulk and vice versa. With our coordinate choice we can now rewrite the DBI action as follows:

$$
S=-\int d^{3} x \sqrt{R^{2}\left(R^{\prime 2}+Z^{\prime 2}\right)-E_{\phi}^{2}\left(R^{\prime 2}+Z^{\prime 2}\right)-R^{2} E_{\theta}^{2}+B^{2}} .
$$

For simplicity we use the DBI action (99) setting the parameters

$$
T_{\mathrm{w}}=1 \text { and } \frac{1}{2 \pi \xi}=1 \text {. }
$$

In the end we will reintroduce the couplings to match our results with the ones of the previous sections. From now on, to simplify the notation, we introduce

$$
l=\sqrt{R^{\prime 2}+Z^{\prime 2}} .
$$

Next, we want to pass from the Lagrangian to Hamiltonian. To this end we must define the canonic momentum conjugated to the electric field,

$$
\Pi^{\theta}=\frac{\delta \mathcal{L}}{\delta E_{\theta}}, \quad \Pi^{\phi}=\frac{\delta \mathcal{L}}{\delta E_{\phi}},
$$


which leads to the following expressions:

$$
\begin{aligned}
& E_{\theta}=\frac{\Pi^{\theta}}{R} \sqrt{\frac{l^{4} R^{2}+l^{2} B^{2}}{l^{2} R^{2}+\Pi^{\phi 2} R^{2}+l^{2} \Pi^{\theta 2}}}, \\
& E_{\phi}=\frac{\Pi^{\phi} r}{l} \sqrt{\frac{B^{2}+l^{2} R^{2}}{\Pi^{\phi 2} R^{2}+l^{2} \Pi^{\theta 2}+l^{2} R^{2}}} .
\end{aligned}
$$

As a result, the Hamiltonian takes the form

$$
\begin{aligned}
\mathcal{H} & =\Pi^{\theta} E_{\theta}+\Pi^{\phi} E_{\phi}-\mathcal{L} \\
& =\frac{1}{l R} \sqrt{\left(B^{2}+R^{2} l^{2}\right)\left(\Pi^{\theta 2} l^{2}+R^{2} l^{2}+\Pi^{\phi 2} R^{2}\right)} .
\end{aligned}
$$

The mass of the solitonic object to be determined is

$$
M=\oint d \theta \oint d \phi \mathcal{H}
$$

with the following conserved charges

$$
n=\oint d \theta \Pi^{\phi}, \quad N=\oint d \phi \Pi^{\theta}, \quad 2 \pi q=\oint d \theta \oint d \phi B .
$$

In the end of this Section we will explain why this is the correct way to relate the charges obtained from the $2+1$ effective action with the ones obtained in the bulk. But for the moment let us stress that the charges $q, n, N$ in (114) are exactly the ones used in Sect. 5.1.

To understand some properties of this toric solution, we have to work in some limit where we are able to solve the problem. Of particular interest is the adiabatic limit in which the radius of the torus $R$ is much bigger than the radius of the tube $\rho$,

$$
l=\sqrt{R^{\prime 2}+Z^{\prime 2}}=\rho \ll R .
$$

In this case we have

$$
\Pi^{\phi}=\frac{n}{2 \pi}, \quad \Pi^{\theta}=\frac{N}{2 \pi}, \text { and } B=\frac{q}{2 \pi},
$$

all independent of $\theta$. The mass of the soliton is

$$
M(\rho, R)=\frac{1}{\rho R} \sqrt{\left(q^{2}+4 \pi^{2} R^{2} \rho^{2}\right)\left(N^{2} \rho^{2}+4 \pi^{2} R^{2} \rho^{2}+n^{2} R^{2}\right)} .
$$


The above expression must be minimized with respect to $\rho$ and $R$. It is clear that such a minimum exists.

Before we pass to minimizing this expression, we can immediately infer some interesting property. First of all, the mass coming from the minimization of (117) is marginally stable with respect to the vertical and horizontal decays. Indeed, under the "vertical" rescaling

$$
(n, N, q) \rightarrow(a n, N, a q)
$$

we have to make a simultaneous rescaling of $M, \rho$ and $R$,

$$
M \rightarrow a M, \quad R \rightarrow R, \text { and } \rho \rightarrow a \rho ;
$$

while under the "horizontal" rescaling

$$
(n, N, q) \rightarrow(n, b N, b q)
$$

we have to make a rescaling of the following form:

$$
M \rightarrow b M, R \rightarrow b R, \text { and } \rho \rightarrow \rho .
$$

Now let us try to minimize the mass function (117). First of all, calculating the derivatives with respect to $R$ and $\rho$, we can get two relations,

$$
R=\frac{1}{\sqrt{2 \pi}} \frac{\sqrt{q N}}{\sqrt[4]{4 \pi^{2} \rho^{2}+n^{2}}}, \quad \rho=\frac{1}{\sqrt{2 \pi}} \frac{\sqrt{q n}}{\sqrt[4]{4 \pi^{2} R^{2}+N^{2}}} .
$$

Next, performing the substitutions $R=a \sqrt{\frac{q N}{n}}$ and $\rho=b \sqrt{\frac{q n}{N}}$ we obtain two equations for $a$ and $b$,

$$
a=\frac{1}{\sqrt{2 \pi} \sqrt[4]{4 \pi^{2} b^{2} \frac{q}{n N}+1}}, \quad b=\frac{1}{\sqrt{2 \pi} \sqrt[4]{4 \pi^{2} a^{2} \frac{q}{n N}+1}} .
$$

Again, as was observed in Sect. 5.1, the solution is governed by the parameter $q /(n N)$. Thus we have to distinguish two regimes.

(i) $q \ll n N$ :

In this regime $a=b=\frac{1}{\sqrt{2 \pi}}$. The radia are

$$
R=\frac{1}{\sqrt{2 \pi}} \sqrt{\frac{q N}{n}}, \quad \rho=\frac{1}{\sqrt{2 \pi}} \sqrt{\frac{q n}{N}} .
$$


while the soliton mass is

$$
M(n, N, q)=2 \sqrt{2 \pi} \sqrt{q n N} .
$$

(ii) $q \gg n N$ :

In this regime

$$
a=b=\frac{1}{(2 \pi)^{7 / 6}} \sqrt[6]{\frac{n N}{q}}
$$

The radia are

$$
R=\sqrt[3]{\frac{1}{4 \pi^{2}} \frac{N^{2} q}{n}}, \quad \rho=\sqrt[3]{\frac{1}{4 \pi^{2}} \frac{n^{2} q}{N}},
$$

while the soliton mass is

$$
M(n, N, q)=2 \pi q
$$

To compare the above results with those of Sect. 5.1 we must reintroduce back the couplings in the action. According to (99) all lengths must be rescaled by the factor $\sqrt[3]{T_{\mathrm{w}}^{-1}}$ while the field strength must be rescaled by the factor $\frac{1}{2 \pi \xi}$. Skipping all passages of the Hamiltonian procedure, we find the new version of the mass formula (117) which reads

$$
\begin{aligned}
M(\rho, R) & =\frac{\Delta m \xi}{\rho R} \sqrt{\frac{1}{4 \pi^{2} \xi^{2}} q^{2}+4 \pi^{2} R^{2} \rho^{2}} \\
& \times \sqrt{\frac{4 \pi^{2}}{\Delta m^{2}} N^{2} \rho^{2}+4 \pi^{2} R^{2} \rho^{2}+\frac{4 \pi^{2}}{\Delta m^{2}} n^{2} R^{2}} .
\end{aligned}
$$

The final expressions for the limiting cases we are interested in are

(i) $q \ll n N$ :

The soliton mass is

$$
M(n, N, q)=4 \pi \sqrt{\xi} \sqrt{q n N}
$$

(ii) $q \gg n N$ :

In this regime the soliton mass is

$$
M(n, N, q)=(\Delta m) q
$$


We note with satisfaction that the mass formulae we have just obtained from the analysis of the DBI action perfectly match our previous results in Sect. 5.1 .

Before concluding this section we have to return to Eqs. (114) and (116) and show that this is indeed the correct way to match the brane and the bulk parameters.

Let us start from the magnetic field. Consider the $Q$ wall studied in Sect. 3.2 with a time-dependent rotation of the phase $\alpha$ given by (32). The $(2+1)$-dimensional magnetic field generated by this rotation is

$$
F_{12}^{(2+1)}=2 \pi \frac{\xi \omega}{\Delta m}
$$

From the bulk standpoint we have an electric field inside the capacitor $E_{z}=$ $\sigma$. From Eq. (44) we have the relation between $\omega$, the phase velocity, and $\sigma$, the charge density on the capacitor. The electric field is thus

$$
E_{z}=\frac{g^{2}}{2} \frac{\omega \xi}{\Delta m}
$$

This justifies the relation (114) for the magnetic field on the brane.

To justify the choice for the electric field conjugates quoted in Eq. (114), we use the ANO vortex ending on the domain wall. From the point of view of the bulk, the vortex is a flux tube carrying $4 \pi n$ units of the magnetic flux. From the brane standpoint the vortex is an electrically charged particle. The electric flux must be measured with the electric displacement $\Pi=\frac{\delta \mathcal{L}}{\delta E}$ which, for small brane curvatures, is

$$
\Pi=\frac{E}{e^{2}}=\frac{E \Delta m}{4 \pi^{2} \xi} .
$$

Using Eq. (23) we can thus express the electric charge as the circulation of the phase $\alpha$ and we finally get

$$
\int \Pi=n
$$

which confirms the first two equation in (114). 


\section{Conclusions and open questions}

Supersymmetric gauge theories are full of surprises and they have now revealed another one: the occurrence of the $Q$ torus and other $Q$ solitons of arbitrary genus! All these solitons are built from a folded domain wall which supports an $\mathcal{N}=2$ Abelian gauge theory on its worldvolume. The domain wall is folded around closed and oriented two-dimensional surfaces. The stabilization of various nontrivial cycles, or handles, is a consequence of magnetic fluxes inside the wall and perpendicular electric fields.

The $Q$ charge obtained by making a time dependent rotation of the $\mathrm{U}(1)$ modulus is crucial for stabilization. After the $Q$-charging procedure the domain wall becomes similar to a capacitor. There are two edges with charge respectively $+q$ and $-q$. Between the two edges there is a Coulomb phase (an instable but stationary point of the potential). At the exterior of the two edges there are respectively vacuum $I$ and vacuum $I I$. In the interior Coulomb phase there are magnetic fluxes that flow along some nontrivial

cycles and cross other nontrivial cycles. For instance, the magnetic flux going around the $\alpha$ cycle passes through the $\beta$ cycle and vice versa. The stabilization of a given cycle occurs through a combination of the magnetic flux that passes through it and the orthogonal electric field created by the $Q$ charging procedure. Both ingredients are equally crucial.

Now it is instructive to comment on the relation between the sigma models, where the toroidal-twisted solitons are stabilized by the Hopf quantum number, and other approaches to $Q$ tori. Clearly, there is a mismatch that must be explained. In the sigma-model limit the soliton is labeled by two numbers, the Hopf charge which we call $k$ (it is the integer invariant of $\pi_{3}\left(\left(S^{2}\right)\right)$, and the $q$ charge. In other approaches we have three numbers, the magnetic fluxes $n$ and $N$ and the $q$ charge. The sigma-model limit, even though it is definitely useful for understanding certain properties of this soliton, looses some information about it. The Hopf charge $k$ is equal to the product of the two magnetic fluxes $n N$. In particular, the sigma-model limit looses the information about the possibility of higher-genus solitons. For higher genera the Hopf charge is

$$
k=\sum_{i=1}^{g} n_{i} N_{i} .
$$

The analysis of $Q$ tori and higher-genus solitons in the bulk theory is very complicated. It is not difficult to write the ansatz for the profile functions and 
for their boundary conditions but a really hard task is to solve the equation of motions which cannot be reduced to ordinary differential equations.

To analyze aspects of this complicated object, we have attacked the problem in a "three-fold" way. The first way is to consider the low-energy effective action on the worldvolume of the domain wall. The second framework is based on the bulk theory in the the limit $\Delta m \ll g \sqrt{\xi}$ where the bulk theory reduces to a nonlinear sigma model $\mathrm{CP}(1)$ and the $Q$ torus is akin to the Hopf Skyrmion. The third way is to consider the bulk theory in the thinedge approximation $\Delta m \gg g \sqrt{\xi}$. In all these three frameworks we proved the existence of $Q$ tori.

An important aspect we should discuss is that of stability of the $Q$-torus. As we have already noted, the issue of the $Q$ charge radiation is essential for understanding whether or not $Q$-torus is a stable object. In fact, it turns out that the only global charge we can measure at infinity is the $Q$ charge. We addressed this question in Sect. 5.6, considering the mass dependence versus $q$ and comparing it with the mass of $q$ elementary quanta. In a number of soliton problems, such as those of $Q$-kink or $Q$-cylinder, we have a powerful argument that answers the question of stability: a lower bound for the energy which is a function of the conserved charges. If the soliton saturates the bound then this is a proof of stability.

Unfortunately, we have no such lower bound for $Q$ tori. In this case the issue of the $Q$ charge radiation must be addressed directly. Analysis of Sect. 5.6 is a step in this direction, but certainly this is not a complete story. First of all, because we consider only certain limits in the parameter space in which we can compute the masses directly. Second, because we should also consider the issue of stability under all kinds of fluctuations around the equilibrium position, in particular, those that break the cylindrical symmetry. This remains to be a task for the future.

In a sense, the $Q$ tori we study are reminiscent of the "vorton" discovered in the context of superconducting cosmic strings [30]. In the latter case we have a string with some internal degrees of freedom; the vorton is a closed and static configuration stabilized by some charge and angular momentum of the flowing degrees of freedom. The question of vorton stability is also a delicate issue [31].

We want to stress that our soliton, although studied in a specific environment of $\mathcal{N}=2$ SQED can appear also in many other contexts. The basic ingredients for building of $Q$ tori and higher-genus solitons of this type are domain walls (or $(2+1)$-dimensional branes) with a $\mathrm{U}(1)$ phase on its 
worldvolume (or, equivalently, a U(1) gauge field). In the the field-theoretic context the theory we studied is the simplest one that supports such kind of solitons. This assertion is easy to understand from the fact that all scalar fields in the theory at hand are excited and play a role in the stability of the $Q$ tori.

The task we had set up is accomplished here. A large number of interesting problems related to supersymmetric $Q$ solitons remain open for future research.

\section{Acknowledgments}

S.B. was funded by the Marie Curie grant MEXT-CT-2004-013510. S.B. wants to thank FTPI for the hospitality in the fall of 2006, when part of this work was done. The work of M.S. was supported in part by DOE grant DE-FG02-94ER408.

\section{References}

[1] M. Shifman and A. Yung, Phys. Rev. D 67, 125007 (2003) hep-th/0212293.

[2] N. Sakai and D. Tong, JHEP 0503, 019 (2005) hep-th/0501207.

[3] A. Hanany, M. J. Strassler and A. Zaffaroni, Nucl. Phys. B 513, 87 (1998) hep-th/9707244].

[4] A. I. Vainshtein and A. Yung, Nucl. Phys. B 614, 3 (2001) hep-th/0012250.

[5] M. Shifman and A. Yung, Supersymmetric Solitons and How They Help Us Understand non-Abelian Gauge Theories, hep-th/0703267.

[6] J. P. Gauntlett, D. Tong and P. K. Townsend, Phys. Rev. D 64, 025010 (2001) hep-th/0012178.

[7] D. Tong, Phys. Rev. D 66, 025013 (2002) hep-th/0202012.

[8] J. P. Gauntlett, R. Portugues, D. Tong and P. K. Townsend, Phys. Rev. D 63, 085002 (2001) hep-th/0008221. 
[9] M. Arai, M. Naganuma, M. Nitta and N. Sakai, Nucl. Phys. B 652, 35 (2003) hep-th/0211103]; BPS wall in $\mathcal{N}=2$ SUSY Nonlinear Sigma Model with Eguchi-Hanson Manifold, in Garden of Quanta, Ed. A. Arafune et. al, (World Scientific, Singapore, 2003) p. 299 hep-th/0302028.

[10] V. A. Novikov, M. A. Shifman, A. I. Vainshtein and V. I. Zakharov, Phys. Rept. 116, 103 (1984).

[11] M. Shifman, Supersymmetric Solitons and Topology, Lect. Notes Phys. 659, 237-284, 2005 (Springer, 2005, Eds. E. Bick and F. Steffen).

[12] A. M. Polyakov, Nucl. Phys. B 120, 429 (1977).

[13] A. Abrikosov, Sov. Phys. JETP 321442 (1957) [Reprinted in Solitons and Particles, Eds. C. Rebbi and G. Soliani (World Scientific, Singapore, 1984), p. 356]; H. Nielsen and P. Olesen, Nucl. Phys. B61 45 (1973) [Reprinted in Solitons and Particles, Eds. C. Rebbi and G. Soliani (World Scientific, Singapore, 1984), p. 365].

[14] G. R. Dvali and M. A. Shifman, Nucl. Phys. B 504, 127 (1997) hep-th/9611213]; G. R. Dvali and M. A. Shifman, in the L. Okun Festschrift, Phys. Rept. 320, 107 (1999) hep-th/9904021.

[15] S. R. Coleman, Nucl. Phys. B 262, 263 (1985), (E) B 269, 744 (1986)].

[16] E. Abraham, Phys. Lett. B 278, 291 (1992); E. R. C. Abraham and P. K. Townsend, Phys. Lett. B 291, 85 (1992); E. R. C. Abraham and P. K. Townsend, Phys. Lett. B 295, 225 (1992).

[17] R. A. Leese, Nucl. Phys. B 366, 283 (1991).

[18] M. Shifman, A. Vainshtein and R. Zwicky, J. Phys. A 39, 13005 (2006) hep-th/0602004.

[19] D. Tong, TASI Lectures on Solitons, hep-th/0509216.

[20] S. Bolognesi, Nucl. Phys. B 730, 127 (2005) hep-th/0507273; Nucl. Phys. B 730, 150 (2005) hep-th/0507286.

[21] S. Bolognesi and S. B. Gudnason, Nucl. Phys. B 741, 1 (2006) hep-th/0512132; Nucl. Phys. B 754, 293 (2006) hep-th/0606065. 
[22] A. M. Polyakov and A. A. Belavin, JETP Lett. 22, 245 (1975).

[23] L. D. Faddeev and A. J. Niemi, Nature 387, 58 (1997) hep-th/9610193.

[24] L. D. Faddeev and A. J. Niemi, Phys. Rev. Lett. 82 (1999) 1624 hep-th/9807069.

[25] S. Bolognesi and M. Shifman, Phys. Rev. D 75, 065020 (2007) hep-th/0701065.

[26] G. H. Derrick, J. Math. Phys. 5, 1252 (1964).

[27] C.V. Johnson, D Branes, (Cambridge University Press, 2003).

[28] D. Mateos and P. K. Townsend, Phys. Rev. Lett. 87 (2001) 011602 hep-th/0103030.

[29] S. Kim, K. M. Lee and H. U. Yee, Supertubes in field theories, hep-th/0603179.

[30] R. L. Davis and E. P. S. Shellard, Phys. Lett. B 209, 485 (1988).

R. L. Davis and E. P. S. Shellard, Nucl. Phys. B 323, 209 (1989).

[31] Y. Lemperiere and E. P. S. Shellard, Phys. Rev. Lett. 91, 141601 (2003) arXiv:hep-ph/0305156. 